\title{
Novel Architecture and Heuristic Algorithms for Software-Defined Wireless Sensor Networks
}

\author{
Ammar Hawbani, Xingfu Wang Member, IEEE, Liang Zhao, Ahmed Al-Dubai Senior Member, IEEE, Geyong \\ Min, Omar Busaileh
}

\begin{abstract}
This paper extends the promising software-defined networking technology to wireless sensor networks to achieve two goals: 1) reducing the information exchange between the control and data planes, and 2) counterbalancing between the sender's waiting-time and the duplicate packets. To this end and beyond the state-of-the-art, this work proposes an SDN-based architecture, namely MINI-SDN, that separates the control and data planes. Moreover, based on MINI-SDN, we propose MINIFLOW, a communication protocol that orchestrates the computation of flows and data routing between the two planes. MINIFLOW supports uplink, downlink and intra-link flows. Uplink flows are computed based on a heuristic function that combines four values, the hops to the sink, the Received Signal Strength (RSS), the direction towards the sink, and the remaining energy. As for the downlink flows, two heuristic algorithms are proposed, Optimized Reverse Downlink (ORD) and Location-based Downlink(LD). ORD employs the reverse direction of the uplink while LD instantiates the flows based on a heuristic function that combines three values, the distance to the end node, the remaining energy and RSS value. Intra-link flows employ a combination of uplink/downlink flows. The experimental results show that the proposed architecture and communication protocol perform and scale well with both network size and density, considering the joint problem of routing and load balancing.
\end{abstract}

Index Terms: wireless sensor networks, heuristic routing, MINI-FLOW, MINI-SDN.

\section{INTRODUCTION}

onventional WSNs have been conceived to be application-specific, which makes it incredibly difficult to reconfigure high-level policies and respond to network-wide events. This is because employing these high-level policies necessitates specifying them in terms of distributed lowlevel configuration [1]. Obviously, task reprogramming or service (e.g., new routing policy) would necessitate each node to be taken out of field-of-interest and the embedded software reprogrammed in the node's hardware [6]. Thus, given the need for large-scale WSNs, this routine would not be realistic due to the fact that the network indeed contains a large number of randomly deployed nodes in a harsh environment that makes it almost impossible to manually take out the nodes after being deployed. Such a problem is inherent to conventional WSNs since each node is manufactured to accommodate all the functionalities from the physical layer to the application layer, behaving like an autonomous system that executes both the data forwarding and the network control [6].

In fact, the application nature in WSN reinforces the need to develop sensor nodes that are characterized as being remotely reconfigurable, reprogrammable, maintainable, and self-healing. By utilizing such sensor nodes, new services and routing policies can be introduced in the network as simple as installing a new software on a PC [3].

Inappropriately, today's conventional WSNs involve

- A. H., XingfuW. \{anmande, wangxfu\}@ustc.edu.cn, and Omar B. (busaileh @mail.ustc.edu.cn) are with School of Computer Science and Technology, University of Science and Technology of China, Hefei, Anhui 230027, China;

- L.Zhao (lzhao@sau.edu.cn); Shenyang aerospace University; Shenyang, 110136, China; Xingfu Wang and Liang Zhao are corresponding authors.

- A.Al-Dubai is with School of Computing; Edinburgh Napier University; (a.al-dubai@napier.ac.uk);

- G. Min is with Department of Computer Science, University of Exeter, Exeter, EX4 4QF, U.K. (e-mail:g.min@exeter.ac.uk); integration and interconnection of many proprietary, vertically integrated sensor nodes that make it extraordinarily rigid to specify high-level network-wide policies using current technologies. The rigidity of underlying infrastructure offers limited options for innovation or improvement since network nodes mostly have been closed with a lowlevel vendor-specific configuration that implements complex high-level network policies [1] [9]. As a result, it is very difficult to replace network nodes with any other produced by any vendor. This curbs the operator in vendor lock-in, and restrains the use of commodity hardware. Besides, it constrains the vendors in manufacturing isolated WSN nodes without adequately reusing common functionalities, which in turn adversely affects production and prototyping [9].

Recent technology shift proposes Software-Defined Networking (SDN) to dramatically simplify network configuration and resource management [7] [4]. By implementing such technology, WSN behavior can be reprogrammed even after the deployment of nodes by remotely injecting embedded defined software into sensor nodes [16]. SDN is essentially featured by disengaging the control decision (control plane) from the network devices, leaving the devices to perform data forwarding functionality (data plane) [8]. Such disengaging is particularly valuable to network operators and designers. In addition, it attracts academia, industry and development community to pay increasing attention to specific research aspects in both WSN and SDN [4].

\subsection{Benefits of SDN in WSN}

1) By employing SDN, the nodes behave as data plane devices to forward data without interfering network control tasks such as topology management and routing strategies, simplifying their architecture and enhancing their energy efficiency. Moreover, armed 
with a network-wide view, the controller introduces comprehensive duty-cycling, scheduling, routing and coverage \& connectivity solutions [5]. Consequently, such sophisticated strategies will be introduced to WSN as simply as it is to remotely install new software on a PC [3].

2) Freeing WSNs from being vendor-specific, proprietary, vertically integrated networks, allowing different vendors to develop application-customizable devices in a non-isolated manner by sharing common functionalities. Besides accelerating the prototyping, production and protocols innovation [2], this avoids the complicated network management imposed by deploying multi-vendor nodes [10].

3) Releasing WSNs from being application-specific, behaving in a plug-and-play manner since the data plane virtually supports all kinds of forwarding rules. Besides, the control plane separates the application layer from the physical layer, supporting multiple applications with different hardware to work under the same physical network architecture [5].

\subsection{Motivations and Contributions}

Based on the limitations of WSNs and the benefits of SDN concept, WSN developers realized the need to shift from conventional WSNs to software-defined WSNs [6]. Recently, a few studies highlighted the importance of extending SDN concepts to WSNs, noticeably focusing on the challenges, the requirements and the logical architecture of network components [2-10]. These studies provided convincing motivations for the extension of SDN concept to conventional WSN. However, these studies mostly addressed the challenges and the abstract architecture while the information exchange between the controllers and the end nodes has not been addressed well so far. Motivated by these observations, in this work, we briefly address the WSN-SDN architecture and then deeply address the flows computation and instantiation, aiming at the following two goals. The first goal is to minimize the information exchange (network overhead) between the controller and the end nodes. The second goal is to trade-off between the waiting-time and the duplicate packets when the nodes are duty-cycled. More details about the trade-off between the sender waiting-time and the duplicate packets can be found in [29]. The main contributions of this paper are as follows:

1) We propose MINI-SDN, a new architecture that integrates the conventional WSN and the Software-Defined Networking (SDN).

2) Based on MINI-SDN, we propose MINI-FLOW, a communication protocol that facilitates the intercommunication between the elements in the data plane and the control plane.

3) We further develop innovative heuristic algorithms to manage uplink, downlink and intra-link flows while reducing the information exchanged between the control and data planes.

4) We implement MINI-SDN \& MINI-FLOW on the platform proposed in [28] [29]. The source code is publicly available in the link ${ }^{1}$. The simulation results showed that our architecture MINI-SDN and communication protocol MINI-FLOW perform and scale well with both network size and density.

The remainder of this paper is organized as follows. The related works are reviewed in the next Section. Section 3 and Section 4 present the MINI-SDN architecture and the MINI-FLOW protocol, respectively. Section 5 provides an analysis of the proposed MINI-FLOW protocol. The experimental results are evaluated in Section 6. Finally, Section 7 concludes this work.

\section{RELATED WORK}

Currently, OpenFlow [18] is the most popular instance of Software-Defined Networking, which has been proposed to resolve analogous issues in wired and address-centric networks. It achieved a high standing position in the networking market and attracted many of the networking leader vendors including $H P, N E C$, NetGear, and IBM, to manufacture OpenFlow-compatible devices available in the market. In addition, it attracted developers to design a variety of SDN-based controllers e.g., NOX, Floodlight, and Maestro, available online [19]. OpenFlow creates one or more Flow Tables for each device to execute the packet lookups and forwarding, controls the communication between the controller and network devices. The flow entry of the Flow Table is composed of three sections, matching rule, actions and statistical information. The matching rule specifies the values and conditions under which the flow entry applies. After matching the rules, the node executes actions (e.g., drop, update, forward to, etc.) identified in the second section of the flow entry. The third section contains statistical information about the flows.

OpenFlow could not fulfill the requirements of WSN as its underlying infrastructure is composed of high-speed switches (e.g., Ethernet/MPLS switches and IP routers) whereas the WSN is characterized by low capabilities in terms of memory, processor, and energy source. Furthermore, WSN is a data-centric network which means that collecting the sensory data is more important than knowing who sent the data, while OpenFlow is designed for addresscentric networks, which implicitly assume the presence of IP-like addressing to create flows.

Technical challenges of extending OpenFlow to WSN are thoroughly articulated in [9]. Motivated by the challenges, including Creating flows, Openflow channel, Overhead of Control Traffic and Traffic Generation, Luo et al. [9] proposed Software-Defined WSN (SD-WSN), an architecture featuring a clear separation between data and control planes. The architecture was divided into three layers, data, control, and application. Moreover, they provided a suite of preliminary solution, named as Sensor OpenFlow (SOF), to overcome the aforementioned challenges. SOF introduces an OpenFlow-extension, supporting SDN-WSN by plugging new forwarding rules to OpenFlow. The rules of SOF slightly meet the requirements of WSN and handle in-network packet processing via various types of WSNs defined addressing. 
Although SOF addressed a few technical challenges of extending SDN to WSN, critical requirements such as duty cycles and in-network data aggregation were not addressed. Therefore, these requirements were analyzed in [4] and based on the discussion, Costanzo et al., proposed an SDN-architecture, named as Software-Defined Wireless Network (SDWN) which is designed based on IEEE 802.15.4 standard (for low-power wireless nodes that operate in 868 $\mathrm{MHz}, 915 \mathrm{MHz}$ and $2.4 \mathrm{GHz}$ frequency bands). Unlike SOF [9], the SDWN [4] contains aggregation layer, data forwarding layer and application layer on the top of PHY and MAC layers. Furthermore, to cope with the challenges and requirements, Smart [10] undertakes a solution to some inherent problems in WSN such as network management, node mobility, localization and topology discovery. Unlike SOF [9] and SDWN [4], Smart [10] composes of five-layer stack, PHY, MAC, NOS (Network Operating System), MW (Middle-Ware) and application layer. Besides, Smart suggests to reside the controller in the sink, and used a localization service for location-based routing.

Furthermore, the requirements such as duty cycles, innetwork data aggregation, flexible rules and space constraints, which are essentials in designing Software Defined Wireless Network, are not considered in OpenFlow. To meet these requirements and inspired by [4] and [9], the WSNWISE [3] [19] extended Openflow to support data aggregation, duty cycling and network function virtualization. It simplifies policy implementation within a reprogrammable and vendor-independent WSN. Besides, WSN-WISE supports multiple controllers serving as a proxy between the two planes, allowing packets to travel following different flow rules defined by different controllers. These controllers periodically update the flow tables to let the nodes know their next-hop node towards the controllers. The topology information is collected through the discovery layer, which has access to the protocol stack. Above the IEEE 802.15.4 stack, WSN-WISE defines the forwarding layer to handle the arriving packets as specified in the Flow Table. On top of the forwarding layer, WSN-WISE defines the In-Network Packet Processing layer to handle data aggregation.

Enabling technologies to implement Software-Defined Sensor Network (SDSN) are presented in [2]. Zeng et al. [2] introduced an SDSNs based Cloud Sensing architecture which consists of a single control server and a set of software-defined nodes. To deploy a new sensing task, the server remotely reprograms a few nodes in a distributed manner such that the reprogrammed nodes are admissible to sense and report the related targets. The functions to be activated in sensor nodes are defined in the server, which provides a role generation and delivery mechanism. Later, SDSN was extended in [16] by proposing a reconfigurable node that consists of a low-power field-programmable gate array (FPGA) and a microcontroller unit (MCU) for changing network behavior.

The rest of this section introduces a few of studies that attempted to address the problems related to the data routing between the two planes, data and control planes. Xiang et al. [25] proposed a routing algorithm in which the nodes are divided into clusters each assigned with a control node (cluster head). Based on the residual energy of the nodes and the transmission distance, the controller selects the control node. The selection of control nodes is formulated as an NP-hard problem which is optimized by adopting particle swarm optimization algorithm. Zeng et al., [26] investigated the coverage sets and nodes activations together with the task assignment and sensing scheduling. These problems are jointly formulated as a mixed-integer with quadratic constraints programming and mixed-integer linear programming. More recently, Li et al. [27] presented a Levenberg-Marquardt algorithm for solving the optimization problem of traffic load. They also provided a convergence analysis of the Levenberg-Marquardt algorithm.

LORA [29] is an opportunistic routing protocol that employed zone routing, in which each node defines a candidate's zone. Candidates within the zone are prioritized based on a metric, which is defined as multiplication of direction, transmission distance distribution and residual energy. ZPR [28] modeled the data routing as an in-zone random process. The zone in ZPR is defined by the source, while the zone in LORA is locally defined by each relay node. Candidates within the zone of ZPR are randomly selected. The implementation of LORA and ZPR is available online in the link ${ }^{2}$ and the link ${ }^{3}$, respectively.

The aforementioned literature provided significant contributions and offered convincing motivations for expanding SDN concepts to WSN. However, designing an abstracted architecture for WSN was their main research point while the information exchange between the controllers and the end nodes has not been addressed well so far. In addition, the combined problem of data routing and load balancing between the two planes is not practically studied. Moreover, the communication overhead between the two planes is not well evaluated. Thus, we go beyond the state-of-the-art by proposing an SDN based architecture called MINI-SDN which separates the control from data plane and paves the way for the proposed communication protocol called MINI-FLOW which undertakes the packet exchange between the two planes.

\section{THE PROPOSED ARCHITECTURE MINI-SDN}

This section explains our proposed software-defined architecture for WSN. As shown in Figure 1, the WSN- SDN logical architecture contains three components, the sensor network (data plane), the sink, and the controller. The nodes reach the sink either directly by one hop or indirectly through multiple hops. The sink acts as an intermediate agent (gateway) between the control plane (controller) and the end nodes (data plane). The controller could be hosted either internally in the sink or externally on a remote server. We consider the controller as an external independent device that communicates with the sink directly but not with the sensor nodes. Like [4], each node in our architecture is equipped with a micro-control unit (IEEE 802.15.4 transceiver). Furthermore, the nodes have limited processing, memory, communication and energy capabilities. The sink transceiver, based on IEEE 802.15.4 standard, is 
connected to an Embedded Network Operation System (ENOS) which is armed with high computing and communication capabilities.

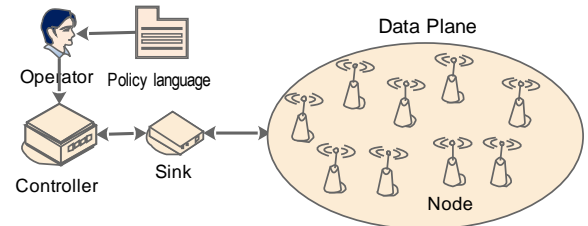

Figure 1: Simplified view of the software-defined sensor network structure.

MINI-SDN is an architecture that separates the control and data planes of WSNs. It consists of three integrated sub-architectures, MINI-SDN-Node, MINI-SDN-Sink and $S D N-W S N$ controller. Figure 2 and Figure 3 explain the proposed architecture of MINI-SDN. Specifically, Figure 2 depicts the proposed architecture for sinks (right side) and the sensor node (left side) while Figure 3 depicts the controller of MINI-SDN. The two architectures (MINI-SDNNode and MINI-SDN-Sink) are intensely explained in the Subsections 3.1 and 3.2, respectively, while the MINI-SDNController is explained in Subsection 3.3.
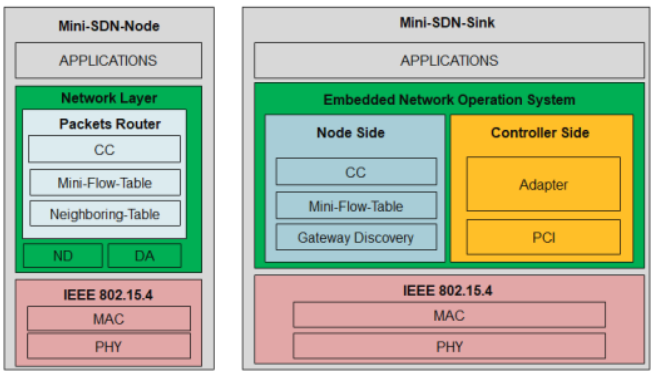

Figure 2: MINI-SDN protocol stack for sink and node.

\subsection{MINI-SDN Architecture for Sensor Nodes}

MINI-SDN-Node protocol stack runs the basic PHY and MAC functionalities defined by the standard IEEE 802.15.4. At the top of IEEE 802.15.4 protocol stack, MINI$S D N-N o d e$ defines the network layer, which runs in the micro-control unit, comprising three main blocks Neighbors Discovery (ND), Data Aggregation (DA), and Packets Router, see Figure 2 (left side). ND allows each node to store and discover the information (i.e., ID, battery state and RSSI) of nearby nodes. To achieve this, each node broadcasts a beacon packet and waits for a response from a nearby node that receives the beacon. $D A$ executes data aggregation or decision fusion, aiming to reduce data redundancy and conserve network resources [9]. Packets Router is adapted to improve the performance of low Duty-cycled WSN by exploiting its broadcast nature (i.e., a node may pick up the packets that are destined to other nodes). Considering the duty cycles where a node randomly switches its mode to active or sleep, the Packets Router selects a set of candidates as potential forwarders in order to reduce sender waiting-time as well as to minimize duplicate packets. When a sender has a packet to send, it transmits preambles continuously until either one of its candidates sends back an ACK or the preset timer of active duration expires. Typically, the sender seizes the earliest forwarding opportunity and does not need to wait for the predefined forwarder to wake up. When multiple candidates wake up, to avoid packet duplication the sender should coordinate its candidates and select one to forward the packet. Packets Router comprises three blocks, Candidates Coordination (CC), MINI-FLOW-Table and Neighboring-Table. Candidates Coordination (CC) implements an important mechanism intended to avoid packets duplication. Given the fact that the nearby nodes can overhear any potential traffic within their ranges, the sender should be able to determine which neighboring node is going to forward the packet to the next hop. Otherwise, a large number of duplicate packets will incur. Note that CC has access to edit the content of MINI-FLOWTable. Packets are forwarded based on the matching rules specified in MINI-FLOW-Table. The rules are remotely computed in the controller. We will deeply explain the MINI-FLOW-Table and Neighboring-Table in Section 4.

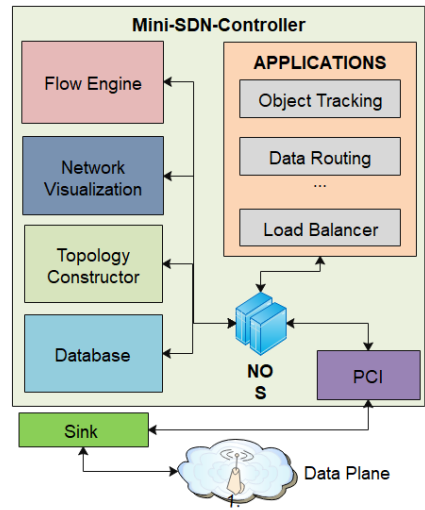

Figure 3: Simplified view of MINI-SDN-Controller

\subsection{MINI-SDN Architecture for Sinks}

The MINI-SDN-Sink architecture is depicted in Figure 2. Like the protocol stack of MINI-SDN-Node explained in the previous subsection, MINI-SDN-Sink runs the basic PHY and MAC functionalities defined by the standard IEEE 802.15.4. At the top of the MAC layer, MINI-SDN-Sink defines Embedded Network Operation System (ENOS), which should have access to all layers including IEEE 802.15.4 protocol stack and application layer, see Figure 2.

The ENOS is an intermediate interface that operates the communications from sink to nodes and from sink to the controller. ENOS involves two main sides, the Controllerside and Node-side. The Node-side is an interface responsible for nodes/sink intercommunications, while the Controllerside is responsible for controller/sink intercommunications. The Node-side contains three parts, the Candidates Coordination (CC), MINI-FLOW-Table, and the Gateway Discovery. The CC and MINI-FLOW-Table implement the same functionalities as in Packets Router of MINI-SDN-Node, explained in the previous subsection. Gateway Discovery discovers the access nodes (i.e., the sensor nodes that directly communicate via one hop with the sink). The Controllerside composes of two components PCI (Programmable Communication Interface) and Adapter. The PCI component is the communication interface (e.g., USB, RS232, TCP/IP, etc.) that manages the communication between the sink and the 
controller while the adapter component is responsible for rendering network policies and messages into a format that can be understood by the sensor nodes and the controller.

\subsection{The Controller of MINI-SDN}

Network services (e.g., high \& low levels network-wide policies, the traffic-forwarding decisions, and so on) are considered as the essential functionality that should be provided by the central controller which employs a Network Operating System (NOS), for example, Linux-based embedded system with high computing and communication capabilities. NOS manages and executes the main network functions such as program execution, I/O operations, security, and communications [13]. Our proposed Mini-Controller for WSN, as shown in Figure 3, implements the network service functions in the NOS, which comprises the Database, Flow-Engine (FE), Network Visualization (NV), Topology Constructor (TC), PCI and Applications. PCI is a communication interface between the controller and the sinks. The Database is designed to store the topological collected data such as the node's ID, node's location, node's neighbors, node's battery level, sinks and so on. Based on the data stored in the Database component, the Topology Constructor constructs a graph-based structure for the sensor network by using the vertices and edges. Utilizing the Topology Constructor and the topological collected data in the Database, the Network Visualization constructs a consistent and comprehensive representation of the current state and statistics of the network. The Flow-Engine is the essential component of the controller in which all the traffic-forwarding decisions are taken and all the high \& low levels network-wide policies are implemented. In addition, FlowEngine translates the network-wide event-driven policies into forwarding rules implemented in the nodes. Besides, it is responsible of updating the MINI-FLOW-Table of the nodes and the sinks.

\section{TABLE 1: NOTATIONS.}

\begin{tabular}{ll}
\hline Notation & Description \\
\hline $\mathbb{N}$ & $\mathbb{N}=\left\{n_{0}, n_{1}, n_{2} \ldots\right\} ; n_{i} \in \mathbb{N}$ is a sensor node. \\
$\mathbb{N}_{i}$ & Neighboring set of $n_{i} ;$ \\
$m_{i}$ & Size of $\mathbb{N}_{i}$. \\
$n_{t}, n_{\mathrm{s}}, n_{\mathrm{b}}$ & End, source and sink nodes, respectively. \\
$H\left(n_{i}\right)$ & Number of hops from $n_{i}$ to the sink. \\
$R\left(n_{j} \in \mathbb{N}_{i}\right)$ & RSSI value from $n_{i}$ to $n_{j} \in \mathbb{N}_{i}$. \\
$L_{i}$ & Residual energy of $n_{i}$. \\
$L_{*}$ & Initial energy. \\
$x_{i}, y_{i}$ & Location of the node $n_{i}$. \\
$e$ & Euler's constant, approximately 2.71828. \\
\hline
\end{tabular}

\section{THE PROPOSED PROTOCOL MINI-FLOW}

MINI-FLOW is designed to manage and compute the flows and the paths between the control and data planes. It involves three mechanisms, the uplink, the downlink and the data routing at the network initialization level. In addition, MINI-FLOW proposes intra-link flows to support routing of the traffic within the data plane. Before addressing these mechanisms, we will first elaborate the substructures of MINI-FLOW, the packet header, packets types, MINI-FLOWTable and Neighbors-Table. By the end of this subsection, we will introduce the forwarders coordination, the update mechanism of the flows and the exponential system parameters that control the flows. Note that, MINI-FLOW is designed based on the low power listening BoX-MAC (i.e., the default MAC protocol in TinyOS) [11]. The common notations used in this paper are summarized in Table 1.

\subsection{Packet Header}

To reach their intended destinations, the packets travel in the network using the fixed header format (10 bytes long) which is similar to the formats mentioned in the literature [3][4] with few differences, the Interested Sinks and the Candidates IDs (see Figure 4). When a network has multiple mobile sinks, the Interested Sinks specifies a group of the sinks which are interested in the content of the packet, whereas the Candidates IDs specifies the Candidates nodes which listened and acknowledged the packet. Furthermore, the header contains the estimated locations of the source and destination. These locations are estimated by utilizing the simplest ranging method, Received Signal Strength (RSS) that represents the relationship between the transmission distance and the signal strength degradation. Such relation is modeled by the path loss of the signal (radio signal degradation with distance). To calibrate the unstable changes of RSS, the self-calibration protocol, explained in [17], is utilized.

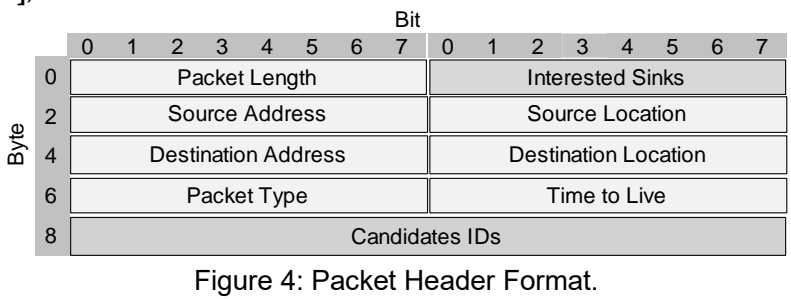

\subsection{Packet Types}

Based on the broadcasting nature of WSNs, MINI-FLOW supports five types of packets, listed as follows.

Type 1. Beacon Packet: the sink or the sensor nodes periodically broadcast this type of packet, which is intended for neighboring discovery, battery state query, etc. The header of this packet does not encapsulate any of the followings: address/location of the destination, Candidates IDs and interested sinks. The time to live is set to one (hop). The nodes, which hear this type of packets, send back a Response Packet to the sender, reporting their information like battery states, number of hops to the sink, ID and RSSI. The header of the Response Packet contains the following: time to live (One hop), Candidates IDs (the sender of beacon packet).

Type 2. Preamble Packet: When a sender has a data packet, it sends preamble packets before transmitting the data packet. The nodes in Neighbors-Table, which hear this type of packet, send back an ACK packet to the sender acknowledging their availability for receiving the data packet. The header of this packet contains the Candidates IDs and the address/location of the sender. It does not contain interested sinks and address/location of the destination. The time to live is set to one (hop).

Type 3. ACK Packet: The nodes in the Neighbors-Table, which hear the Preamble Packet, send back an ACK packet to 
the sender acknowledging their availability for receiving the data packet. When the sender receives the ACK packet, it sets the $A C K$ value (Table 2) to 1.0 .

Type 4. Data Packet: After receiving an ACK-Packet, the Candidates Coordination (CC) in the Packets Router (Figure 2) sends the data packet to the next hop based on the Priority value (Table 2). In this type of packet, the address/location of destination are attached to the header. In the field of Candidates IDs, the sender node specifies one candidate (next hop in the path) based on the Priority (Table 2).

Type 5. Control Packet: The exchanged packets between the controller and the nodes include the rule/action request, rule/action response, topology info, etc.

\subsection{MINI-FLOW-Table}

Broadcasting nature and resource scarcity in WSNs impose that the WSN-SDN architecture should be characterized by being simplified and highly efficient. In such constrained networks, the nodes periodically switch between active and sleep states according to predefined wake-up intervals. MINI-FLOW is designed based on the low power listening BoX-MAC. MINI-FLOW saves the flows as the entries in the MINI-FLOW-Table, shown in Table 2, in which each flow is defined based on the direction of the flow, uplink, downlink or intra-link.

TABLE 2: MINI-FLOW-TABLE.

\begin{tabular}{|c|c|c|c|c|c|}
\hline Candidate ID & Flow-Direction & Priority & ACK & Action & Statistics \\
\hline 1 & Uplink & 0.37 & 1 & Forward & 37 \\
\hline 2 & Uplink & 0.34 & 0 & Forward & 34 \\
\hline 3 & Uplink & 0.29 & 1 & Drop & 29 \\
\hline 1 & Downlink & 0.20 & 1 & Drop & 20 \\
\hline 2 & Downlink & 0.30 & 1 & Drop & 30 \\
\hline 3 & Downlink & 0.50 & 0 & Forward & 50 \\
\hline 1 & Intra-Link & 0.46 & 0 & Forward & 48 \\
\hline 2 & Intra-Link & 0.44 & 1 & Forward & 45 \\
\hline 3 & Intra-Link & 0.10 & 1 & Drop & 7 \\
\hline
\end{tabular}

MINI-FLOW-Table is designed to achieve two goals. The first goal is to reduce the exchange of information between the controller and end nodes by using a fixed flow that targets each node rather than each packet. The controller delivers the uplink, downlink or intra-link flows to each node after network initialization. The controller does not need to define the flows for each packet. This reduces the amount of information exchanged between the two planes. The controller updates the flows directly based on the statistics received from the end nodes. Flows could be updated whenever an end-node requests the routing policy.

TABLE 3: NEIGHBORS-TABLE.

\begin{tabular}{|c||c|c||c|c|}
\hline Node ID & Battery Level & Estimated Location & $\mathrm{H}(\mathrm{i})$ & $\mathbf{R}(\mathrm{i})$ \\
\hline $\mathrm{n} 1$ & $\mathrm{Li}, 1$ & $\mathrm{x} 1, \mathrm{y} 1$ & $\mathrm{Hi}, 1$ & $\mathrm{Ri}, 1$ \\
\hline $\mathrm{n} 2$ & $\mathrm{Li}, 2$ & $\mathrm{x} 2 \mathrm{y2}$ & $\mathrm{Hi}, 2$ & $\mathrm{Ri}, 2$ \\
\hline $\mathrm{n} 3$ & $\mathrm{Li}, 3$ & $\mathrm{x} 3, \mathrm{y} 3$ & $\mathrm{Hi}, 3$ & $\mathrm{Ri}, 3$ \\
\hline $\mathrm{n} 4$ & $\mathrm{Li}, 4$ & $\mathrm{x} 4, \mathrm{y} 4$ & $\mathrm{Hi}, 4$ & $\mathrm{Ri}, 4$ \\
\hline
\end{tabular}

The second goal is, in case of duty-cycled nodes, to reduce the waiting-time and to minimize the duplicate packets, each node is assigned with multiple flows each has a Priority value, which is heuristically computed in the Flow-Engine. When the sender-node has a packet, it sends a preamble packet, which will be heard by all awaken neighboring nodes in Table 3. The nodes, which received the preamble packet, will send back an ACK to the sender. The sender sets the $A C K$ value to 1.0 in the MINI-FLOW-Table. Otherwise, the $A C K$ value is set to 0.0. Based on the Priority value, if the flow is matched (i.e., the candidate is selected), then the corresponding Action is executed and the information in the Statistics is updated. The Statistics information is periodically reported to the controller. When the ACK value is 0.0, the corresponding Action will not be executed even though the Priority value is the highest. Table 2 shows an example of the flows in a node that has three neighboring nodes. We will explain how the controller computes the Actions and Priority in the Subsection 4.4. Table 3 shows the information to be stored in the node $n_{i}$. We assume it has four neighboring nodes $\mathbb{N}_{i}=\left\{n_{1}, n_{2}, n_{3}, n_{4}\right\}$. The function $H\left(n_{j} \in \mathbb{N}_{i}\right)$ returns the number of hops from $n_{j}$ to the sink while $R\left(n_{j} \in \mathbb{N}_{i}\right)$ returns the RSSI value from $n_{i}$ to $n_{j}$.

\subsection{Flows Computing}

This section is devoted for computing uplink flows (i.e., the paths from the end nodes to the controllers), the downlink flows (i.e., the paths from the controllers to the end nodes), and the intra-link flows (i.e., data traffic within the data plane such as a path from a node to a cluster head $\mathrm{CH}$ in clustered WSN). MINI-FLOW supports both flat-based routing (i.e., all nodes of equal role) and hierarchical-based routing (i.e., different nodes may assume different roles). The following four subsections (4.4.1 to 4.4.4) elaborate the flows of flat-based routing while Subsection 4.4.5 explains the flows of hierarchical-based routing.

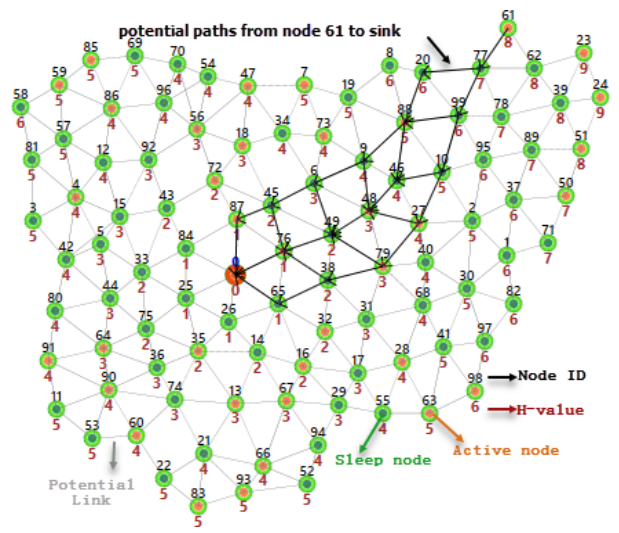

Figure 5: Network initialization. An example of 100 nodes (indicated by the circle). The digits indicate the $\mathrm{H}$-value (lower) and the ID (upper) of each node. The arrows show the available paths from the source node $n_{61}$ to the $\operatorname{sink} n_{0}$. H-value is computed by ALGORITHM 1.

\subsubsection{Network Initialization}

The goal of this phase is to have each node identify its $H$ value(s), so that it can simply share the information (i.e., $R$ values, $H$-value and energy level) with the controller. In flatbased routing, the initialization phase starts at the sink where the $H$-value is set to 0 . The sink starts the initialization by sending a beacon, then the nodes that received the beacon execute ALGORITHM 1, and further send their own new beacon. The process is terminated when each node knows its $\mathrm{H}$-value. Figure 5 shows an example of implementing ALGORITHM 1 on 100 nodes. The collected information is reported to the sink by the following four steps. (1) The sender node transmits a preamble packet. (2) The nodes that received the preamble packet send back the ACK 
packets to the sender expressing their availability. (3) The Packets Router selects a candidate from the nodes, which expressed their availability providing that the $\mathrm{H}$-value of the candidate node is smaller than the $\mathrm{H}$-value of the sender. (4) The Packets Router specifies the ID of the candidate in the Candidates IDs field of the Control Packet. This process is repeated until the Packet reaches the sink node. The collected information is stored in the Database so the Network Visualization (NV), Topology Constructor (TC) and Flow-Engine (FE) can utilize it. In addition, each node saves its neighbors information in the Neighbors-Table (Table 3).

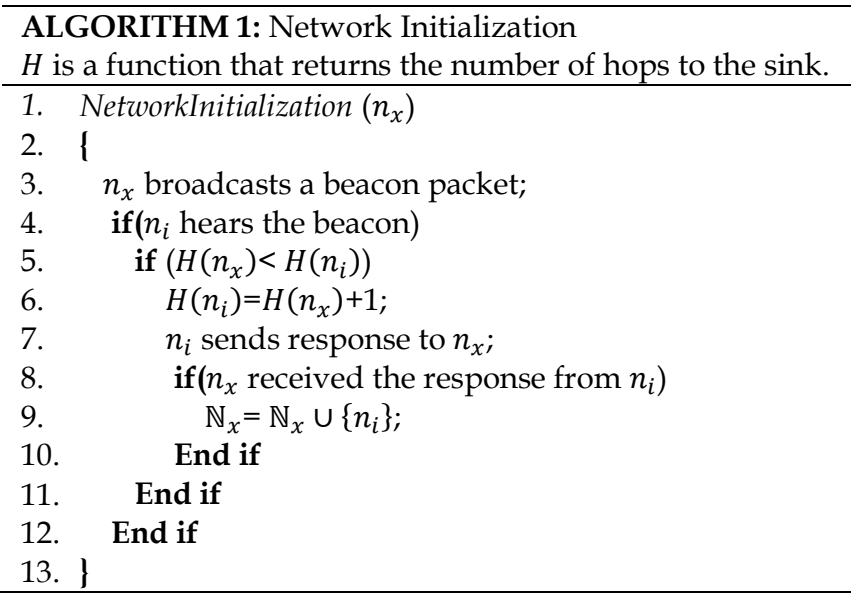

\subsubsection{Uplink Flows}

Uplink flows are computed through a heuristic function that combines four values, the number of hops to the sink, the Received Signal Strength (RSS), the direction towards the sink and the remaining energy. The data routing from nodes to the controllers is determined based on the Priority value of each flow in the MINI-FLOW-Table (Table 2). As shown in Figure 5, each node has multiple candidates at each transmission stage. Accordingly, each node has multiple potential paths to reach the sink. To optimize the path selection, we propose a heuristic algorithm that depends on the four values $H$-value, $R$-value, $\mathcal{E}$-value and L-value. $\mathcal{E}$ value represents the Euclidean distance from the node to the sink. H-value represents the number of hops from the node to the sink, obtained by ALGORITHM 1. L-value represents the remaining energy while $R$-value is a function of the distance between the sender and receiver node, which varies due to various in-path interferences [12]. $R$-value is considered as a term in the proposed heuristic function since the energy consumption is strongly related to the transmission distance between the sender and receiver nodes [14]. Each of the four values has an impact on path selection.

(1) H-Distribution: This probability distribution prioritizes the nodes, which have smaller $H$-value (i.e., minimum the number of hops to the sink as computed by ALGORITHM 1). Each node $n_{i}$ expresses the $H$-values of its neighboring nodes $\mathbb{N}_{i}$ as a vector, $\mathcal{H}_{i}=\left\{H_{i, 1}, H_{i, 2}, \ldots, H_{i, m_{i}}\right\}$ where $H_{i, j}=H\left(n_{j}\right), n_{j} \in \mathbb{N}_{i}, m_{i}=\left|\mathbb{N}_{i}\right|$. Then, $\mathcal{H}_{i}$ is normalized into $\overline{\mathcal{H}}_{i}=\left\{\bar{H}_{i, 1}, \bar{H}_{i, 2}, \ldots, \bar{H}_{i, m_{i}}\right\}$ by Eq.(1). Finally, the $H$ value Distribution is defined by the mass function Eq.(2), denoted by $\widetilde{\mathcal{H}}_{i}=\left(\widetilde{H}_{i, 1}, \widetilde{H}_{i, 2}, \ldots, \widetilde{H}_{i, m_{i}}\right)$. Note that in Eq.(1), the $\gamma_{H} \geq 0$ is called the H-exponent. Greater value of $\gamma_{H}$ offers a higher probability distribution for the nodes with smaller
$H$-values to be selected as candidate nodes.

$$
\begin{array}{r}
\bar{H}_{i, j}=\left(1+H\left(n_{j}\right)\right)^{-\gamma_{H}} \forall n_{j} \in \mathbb{N}_{i}(1) \\
\widetilde{H}_{i, j}=\left(1-e^{\bar{H}_{i, j}}\right) / \sum_{k=1}^{m_{i}}\left(1-e^{\bar{H}_{i, k}}\right) \forall n_{j} \in \mathbb{N}_{i}
\end{array}
$$

Figure 6 shows the impact of $\gamma_{H}$ on H-Distribution when node $n_{i}$ has 5 neighbor nodes, $\mathbb{N}_{i}=\left\{n_{1}, n_{2}, \ldots, n_{5}\right\}$. For simplicity, the $H$-Values of the neighboring nodes are assumed to be $\mathcal{H}_{i}=\left\{H_{i, 1}=1, H_{i, 2}=2, \ldots, H_{i, 5}=5\right\}$, whereas $\gamma_{H}$ varies from 0 to 1 . The $H$-Distribution curve shows that the larger the value of $H$-exponent $\left(\gamma_{H}\right)$, the greater the probability of selecting the node having a smaller $\mathrm{H}$-value.

(2) R-Distribution: This distribution prioritizes the node with larger $R$-value. We assume that the RSSI value is represented in a positive form, the closer the value is to zero, the stronger the received signal has been. RSSI can vary greatly and affect the functionality in wireless networking. It is derived in the intermediate frequency (IF) stage before the IF amplifier. Node $n_{i}$ expresses the $R$-values of its neighbor nodes $\mathbb{N}_{i}$ as a vector $\mathcal{R}_{i}=$ $\left\{R_{i, 1}, R_{i, 2}, \ldots, R_{i, m_{i}}\right\} \quad$ where $R_{i, j}=R\left(n_{j}\right), n_{j} \in \mathbb{N}_{i}, m_{i}=\left|\mathbb{N}_{i}\right|$. Then, $\mathcal{R}_{i}$ is normalized into $\overline{\mathcal{R}}_{i}=\left\{\bar{R}_{i, 1}, \bar{R}_{i, 2}, \ldots, \bar{R}_{i, m_{i}}\right\}$ by Eq.(3). Finally, the R-value Distribution is defined by the mass function Eq.(4), denoted by $\tilde{\mathcal{R}}_{i}=\left(\tilde{R}_{i, 1}, \tilde{R}_{i, 2}, \ldots, \tilde{R}_{i, m_{i}}\right)$. Note that in Eq.(4), the $\gamma_{R} \geq 0$ is called the $R$-exponent. Larger value of $\gamma_{R}$ offers higher probability for the nodes, which have greater $R$-value to be selected as forwarders.

$$
\begin{gathered}
\bar{R}_{i, j}=1-\left(\left[R\left(n_{j}\right)^{\gamma_{R}}\right] / \sum_{k=1}^{m_{i}} R\left(n_{k}\right)\right) \forall n_{j} \in \mathbb{N}_{i} \text { (3) } \\
\tilde{R}_{i, j}=e^{\bar{R}_{i, j}} / \sum_{k=1}^{m_{i}} e^{\bar{R}_{i, k}} \forall n_{j} \in \mathbb{N}_{i}
\end{gathered}
$$

During simulation experiments, RSSI-based distance estimation model, as explained in [15], is employed to obtain the expected transmission distance. We implemented the free space propagation model [24] that assumes the ideal propagation condition by representing the communication range as a circle around the transmitter. If a receiver is within the circle, it receives all packets. Otherwise, it loses all packets [17]. Figure 7 shows the impact of $R$ - exponent $\gamma_{R}$ on the R-Distribution ( $\tilde{\mathcal{R}}$ ) when $n_{i}$ has 5 neighbor nodes $\mathbb{N}_{i}=\left\{n_{1}, n_{2}, \ldots, n_{5}\right\}$ with transmission distance varying from $50 \mathrm{~m}$ to $250 \mathrm{~m}$. The $R$-Distribution Control $(R C)$ varies from 0 to 1 . The $R$-Distribution curve shows that as the value of $\gamma_{R}$ becomes larger, the probability of selecting the nodes having larger $R$-value becomes higher.

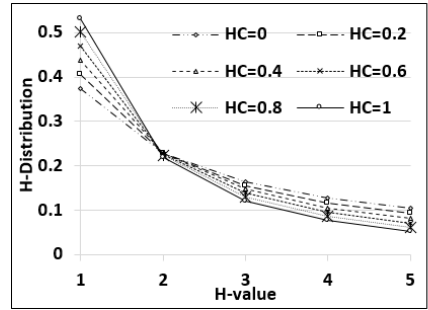

Figure 6: The curve of $\mathrm{H}$-Distribution when the $\mathrm{H}$-exponent control (HC) varies from 0.0 to 1.0

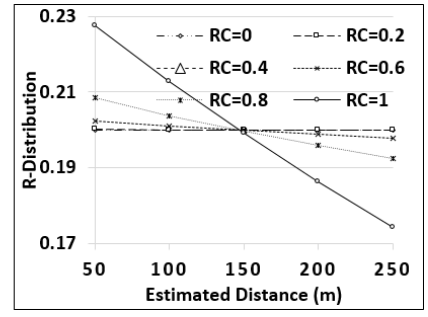

Figure 7: The curve of R-Distribution when the R-exponent control $(R C)$ varies from 0.0 to 1.0
(3) L-Distribution: The goal of this distribution is to assign a higher priority to the nodes with a greater remaining 
energy level. Node $n_{i}$ defines an energy-normalized vector $\overline{\mathcal{L}}_{i}=\left(\bar{L}_{i, 1}, \bar{L}_{i, 3}, \ldots, \bar{L}_{i, m_{i}}\right)$ by Eq.(5). Then, the values on vector $\overline{\mathcal{L}}_{i}$ are plugged into the mass function Eq.(6) to obtain a discrete random variable, $\tilde{\mathcal{L}}_{i}=\left(\tilde{L}_{i, 1}, \tilde{L}_{i, 2}, \ldots, \tilde{L}_{i, m_{i}}\right)$. Note that $L_{j}$ denotes the residual energy of node $n_{j}$ while $L_{*}$ denotes the initial energy of $n_{j}$. Also, in Eq.(6), the $\gamma_{\mathrm{L}} \geq 0$ is called the L-exponent. Greater value of $\gamma_{L}$ offers greater probability distribution for the nodes, which have more remaining energy to be selected as candidates.

$$
\begin{gathered}
\bar{L}_{i, j}=\left[\frac{L_{j}}{L_{*}}\right]^{\gamma_{L}} \quad \forall n_{j} \in \mathbb{N}_{i} \\
\tilde{L}_{i, j}=\left(1-e^{-\bar{L}_{i, j}}\right) / \sum_{k=1}^{m_{i}}\left(1-e^{-\bar{L}_{i, k}}\right) \quad n_{j} \in \mathbb{N}_{i} ; \gamma_{L} \geq 0
\end{gathered}
$$

Figure 8 shows the impact of L-exponent $\left(\gamma_{\mathrm{L}}\right)$ on the $L$ Distribution $\left(\tilde{\mathcal{L}}_{i}\right)$ when node $n_{i}$ has 5 neighbor nodes $\mathbb{N}_{i}=$ $\left\{n_{1}, n_{2}, \ldots, n_{5}\right\}$. For simplicity, the L-Values of the neighboring nodes are assumed to be $\mathcal{L}_{i}=\left\{L_{i, 1}=10, L_{i, 2}=\right.$ $\left.20, \ldots, L_{i, 5}=50\right\}$ whereas $\gamma_{L}$ varies from 0 to 1 . The $L$-Distribution curve revealed that when the value of $L$-exponent $\gamma_{L}$ is greater, the probability of selecting the nodes which have more residual energy (L-value) gets higher.

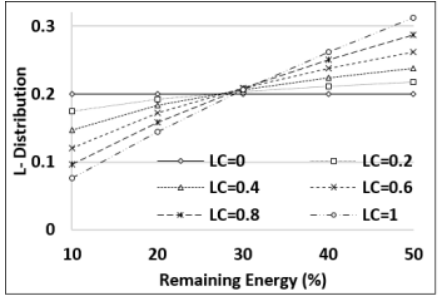

Figure 8: The curve of L-Distribution when the L-exponent $\gamma_{L}$ varies from 0.0 to 1.0 .

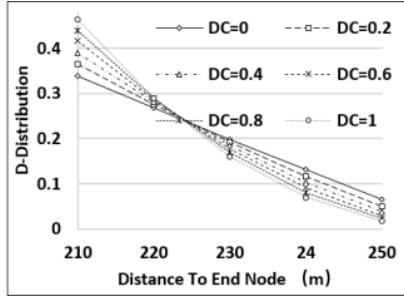

Figure 9: The curve of D-Distribution when the $D$-exponent control (DC) varies from 0.0 to 1.0 .
(4) Euclidean Distance to the Sink (E-Distribution): The $\mathcal{E}$-Distribution aims to assign a higher priority in each transmission session for the nodes that are in the direction towards the $\operatorname{sink} n_{b}$. Sender $n_{i}$ expresses the $\mathcal{E}$-value of its neighbor nodes (i.e., $\forall n_{j} \in \mathbb{N}_{i}$ ) as a vector, $\varepsilon_{i}=$ $\left\{\varepsilon_{i, 1}, \mathcal{E}_{i, 2}, \ldots, \mathcal{E}_{i, m_{i}}\right\}$, where $\mathcal{E}_{i, j}$ represents Euclidean distance from relay node $n_{j}$ to sink $n_{b}$ as expressed in Eq. (7). The vector $\varepsilon_{i}$ is normalized into $\bar{\varepsilon}_{i}=\left\{\bar{\varepsilon}_{i, 1}, \bar{\varepsilon}_{i, 2}, \ldots, \bar{\varepsilon}_{i, m_{i}}\right\}$ by Eq. (8), where $\varphi_{i}$ denotes the communication radius of $n_{i}$. Finally, the $\mathcal{E}$-Distribution is obtained by Eq. (9), $\tilde{\varepsilon}_{i}=$ $\left(\tilde{\varepsilon}_{i, 1}, \tilde{\varepsilon}_{i, 2}, \ldots, \tilde{\varepsilon}_{i, m_{i}}\right)$. In Eq. (8), $\gamma_{\varepsilon} \geq 0$ is a system parameter, called the $\mathcal{E}$ - exponent. Greater value of $\gamma_{\varepsilon}$ offers a higher probability for the nodes that have smaller $\mathcal{E}$-value (closer to the sink node) to be selected as candidates.

$$
\begin{aligned}
& \mathcal{E}_{i, j}=\sqrt{\left(x_{b}-x_{j}\right)^{2}+\left(y_{b}-y_{j}\right)^{2}} \quad n_{j} \in \mathbb{N}_{i} \\
& \bar{\varepsilon}_{i, j}=\left(1-\sqrt{\frac{\varepsilon_{i, j}}{\varepsilon_{i, b}+\varphi_{i}}}\right)^{\gamma_{\varepsilon}}, n_{j} \in \mathbb{N}_{i} \\
& \tilde{\varepsilon}_{i, j}=\bar{\varepsilon}_{i, j} / \sum_{k=0}^{m_{i}} \bar{\varepsilon}_{i, k}, n_{j} \in \mathbb{N}_{i}
\end{aligned}
$$

(5) Uplink Flow Priority: The uplink Priority is computed by the heuristic function defined as the average sum of the four distributions, $H$-Distribution, $R$-Distribution, $\mathcal{E}$ Distribution and L-Distribution as formulated in Eq. (10). The four probability distributions are considered as four forces controlling the forwarding process at the same time. Each force has a different impact on the forwarding process, so the greater the force, the greater the influence on the forwarding process. The Priority value $\tilde{\mathcal{P}}_{i, j}$ indicates the probability of transmitting the data packet from the sender $n_{i}$ to the receiver $n_{j}$ in the flow entries (Table 2).

$$
\begin{gathered}
\widetilde{\mathcal{P}}_{i, j}=\left(\widetilde{H}_{i, j}+\tilde{R}_{i, j}+\tilde{L}_{i, j}+\tilde{\varepsilon}_{i, j}\right) / 4 \\
=\frac{1}{4}\left[\left(1-e^{\bar{H}_{i, j}}\right) / \sum_{k=1}^{m_{i}}\left(1-e^{\bar{H}_{i, k}}\right)+e^{\bar{R}_{i, j}} / \sum_{k=1}^{m_{i}} e^{\bar{R}_{i, k}}+\left(1-e^{-\bar{L}_{i, j}}\right) / \sum_{k=1}^{m_{i}}\left(1-e^{-\bar{L}_{i, k}}\right)\right. \\
\left.+\bar{\varepsilon}_{i, j} / \sum_{k=0}^{m_{i}} \bar{\varepsilon}_{i, k}\right] n_{j} \in \mathbb{N}_{i}(10)
\end{gathered}
$$

\subsubsection{Downlink Flows}

We propose two heuristic algorithms for the downlink. The first algorithm simply considers the downlink flows as the reverse of the uplink flows. The paths should be specified in the header of the packet. In the second algorithm, the paths are computed based on a heuristic function that considers three values, distance to the end node, the remaining energy and RSSI value. The location of the end node is specified in the header of the packet. The two heuristic algorithms are precisely clarified in the following subsections.

(1) Optimized Reverse Downlink (ORD): The paths from the controller to end nodes are locally computed in the controller rather than in each node. There are multiple reverse paths from the controller to each end node (e.g., see Figure 10, the reverse paths from the sink node $n_{0}$ to the end node $n_{61}$ ). To optimize the downlink selection, based on the paths computed in the initialization process, the controller constructs a sub-graph $G_{i}=\left(V_{i}, E_{i}\right)$ for the target end node $n_{t}$ and applies both the $R$-value and the L-value based on the collected information in the database. The priority of each potential path is computed as follows. Let $\mathbb{P}_{t}^{b}=$ $\left\{n_{b}, \ldots, n_{t}\right\}$ be a path from the sink node $n_{b}$ to the end node $n_{t}$. The ordered pair $\left(n_{i}, n_{j}\right)$ represents one hop from $n_{i}$ to $n_{j}$ while the set $E\left(n_{i}\right)$ represents the adjacent edges of $n_{i}$. The priority of the path $\mathbb{P}_{t}^{b}$, denoted by $\widetilde{\mathbb{P}}_{t}^{b}$, is computed by Eq. (11) where $\tilde{R}_{i, j}$ and $\tilde{L}_{i, j}$ are computed by Eq. (4) and Eq. (6), respectively. The controller sorts the obtained potential paths according to their priority. Higher priority implies higher path utility.

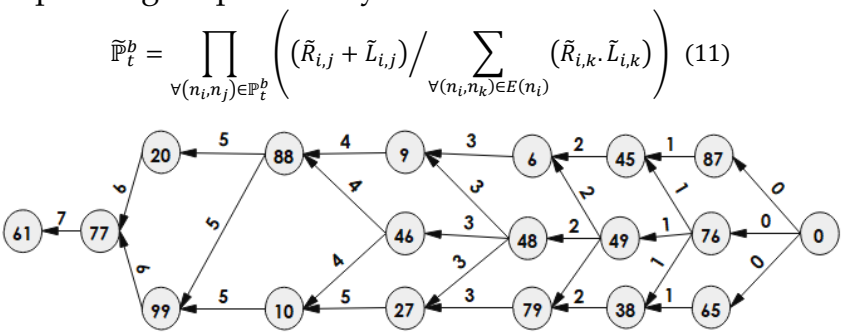

Figure 10: The Sub-graph of the end node $n_{61}$. It shows the potential reverse paths from the sink $n_{0}$ to the node $n_{61}$ (the network topology is depicted in Figure 5). Each path contains eight hops. The weight of the edge (link) indicates the $H$-value (obtained by ALGORITHM 1 ) while the vertex ID indicates the ID of the sensor node.

(2) Location-based Downlink (LD) Algorithm: considering the values which have an influence on the selection of the path from the controller to the end node, the priority of the downlink is computed as an average sum of three 
values, $R$-value, $D$-value (the distance to the end node) and $L$-value. The R-Distribution and L-Distribution are explained in the previous subsection while the D-Distribution is explained below.

(a) Distance to End Node (D-Distribution): The goal of D-Distribution is to allocate a higher priority for the relay nodes that are closer to the end node $n_{t}$. The sender node $n_{i}$ expresses the $D$-values of its neighbor nodes $\forall n_{j} \in$ $\mathbb{N}_{i}$ as a vector, $\mathcal{D}_{i}=\left\{D_{i, 1, t}, D_{i, 2, t}, \ldots, D_{i, m_{i}, t}\right\}$ where $D_{i, j, t}=D_{j, t}$ denotes the Euclidean distance from the relay node $n_{j}$ to the end node $n_{t}$, expressed in Eq. (12). $\mathcal{D}_{i}$ is normalized into $\overline{\mathcal{D}}_{i}=\left\{\bar{D}_{i, 1, t}, \bar{D}_{i, 2, \mathrm{t}}, \ldots, \bar{D}_{i, m_{i}, t}\right\}$ by Eq. (13) where $\varphi_{i}$ is the communication radius of $n_{i}$. Finally, the D-Distribution is defined by the mass function Eq. (14), denoted by $\widetilde{\mathcal{D}}_{i}=$ $\left(\widetilde{D}_{i, 1, t}, \widetilde{D}_{i, 2, t}, \ldots, \widetilde{D}_{i, m_{i}, t}\right)$. In Eq. (14), $\gamma_{D} \geq 0$ is called the Dexponent. Greater $\gamma_{D}$ offers a higher probability for the nodes, which have smaller $D$-value (closer to the end node) to be selected as candidates. Figure 9 shows the impact of $\gamma_{D}$ on the D-Distribution when $n_{i}$ has 5 neighbor nodes $\mathbb{N}_{i}=\left\{n_{1}, n_{2}, \ldots, n_{5}\right\}$. The distance from $n_{i}$ to $n_{t}$ is $225 \mathrm{~m}$ while the distances from the neighbor nodes to $n_{t}$ are expressed by $\mathcal{D}_{i}=\left\{D_{i, 1, t}=210, D_{i, 2, \mathrm{t}}=220, \ldots, D_{i, 5, t}=250\right\}$. The value of $\gamma_{D}$ varies from 0 to 1 . The curve of the $D$-Distribution shows that as the value of D-exponent $\left(\gamma_{D}\right)$ becomes larger, the probability of selecting the nodes closer to $n_{t}$ becomes higher.

$$
\begin{aligned}
& D_{i, j}=\sqrt{\left(x_{i}-x_{j}\right)^{2}+\left(y_{i}-y_{j}\right)^{2}} \\
& \bar{D}_{i, j, t}=\left(\frac{D_{j, t}}{D_{i, t}+\varphi_{i}}\right), n_{j} \in \mathbb{N}_{i} \\
& \widetilde{D}_{i, j, t}=\left(1-\sqrt{\bar{D}_{i, j, t}}\right)^{1+\gamma_{D}} / \sum_{k=0}^{m_{i}}\left(1-\sqrt{\bar{D}_{i, k, t}}\right)^{1+\gamma_{D}}, n_{j} \in \mathbb{N}_{i}
\end{aligned}
$$

(b)Downlink Flow Priority: The three distributions (quantities), R-Distribution, D-Distribution and L-Distribution, are considered as three forces that control the downlink flow. The impacts of these forces are tuned by the exponential parameters such that a greater value of the exponent increases the impact of the force. Consequently, to counterbalance the impact of these forces, the Priority of the downlink is computed as an average sum of these three forces, formulated in Eq. (15).

$$
\begin{gathered}
\tilde{\mathcal{P}}_{i, j, t}=\left(\tilde{R}_{i, j}+\widetilde{D}_{i, j, t}+\tilde{L}_{i, j}\right) / 3 \\
\left.\left.+\left(1-e^{-\bar{L}_{i, j}}\right) / \sum_{k=1}^{m_{i}}\left(1-e^{-\bar{L}_{i, k}}\right)\right], n_{j} \in \sum_{k=1}^{\bar{H}_{i, j}}\right) / \mathbb{N}_{i}
\end{gathered}
$$

\subsubsection{Intra-Link Flows}

In hierarchical-based routing e.g., clustered-based, the members hand the collected data to the $\mathrm{CH}$ which then delivers it further to the sink directly or by another $\mathrm{CH}$ (multihops). MINI-FLOW instantiates the intra-link flows to support the data traffic within the data plane devices. The initialization of the network in hierarchical-based routing starts at a $\mathrm{CH}$ where the $\mathrm{H}$-value is set to 0 . The $\mathrm{CH}$ starts the initialization phase by sending beacons. The nodes that receive the beacon within the cluster execute ALGORITHM 1 and further send their new beacons. The uplink/downlink flows from/to the $\mathrm{CH}$ to/from the sink in hierarchical-based routing are computed exactly as in flat-based routing. Similarly, the intra-link flows from the end node to the $\mathrm{CH}$ can be computed based on a heuristic function that combines four values, the number of hops to the $\mathrm{CH}$, the Received Signal Strength (RSS), the direction towards the $\mathrm{CH}$ and the remaining energy. Similar to the downlink, the intra-link from $\mathrm{CH}$ to the end node is computed as an average sum of the three values, $R$-value, D-value (the distance from $\mathrm{CH}$ to the end node) and L-value.

\subsection{Candidates and Coordination}

Commonly, the MAC layer coordinates the active/sleep states by one of the two approaches: synchronous or asynchronous [21]. In the synchronous approach, the MAC protocol synchronizes the wake-up intervals and active periods such that multiple nodes wake up at the same time and have the same active period. While in the asynchronous approach, the nodes randomly define their wake up intervals and active periods [23]. Sender and receiver nodes should be involved in an active state in order to undertake the communication task. This roughly runs in three steps [22]. First, the Network Layer determines the next hop (forwarder) using a predefined routing metric e.g., link quality, number of hops, etc. Second, the MAC Layer waits for the intended forwarder to wake up and receive the packet. Third, the forwarder sends back an ACK to the sender, acknowledging the received packet. These requirements are neglected in OpenFlow wired domains. Coordination among candidates is an important mechanism to avoid the negative impact of packets duplication. When more candidates (neighboring nodes in active mode) have received the preamble packet, the next step is to ensure that the data packet will be sent to one candidate. The Candidates Coordination (CC) selects the node, which has the maximum Priority in Table 2. The number of candidates in uplink and downlink are controlled by network density in a distributed manner.

Uplink-Flow Action: The action of the uplink flow, which must be executed on the packets (i.e., drop, forward), is defined by Eq. (16) where $\vec{C}_{i}$ is the threshold of uplink candidates that are allowed to be selected by the node $n_{i}$.

$$
\overrightarrow{A c t}\left(n_{i}\right)=\left\{\begin{array}{l}
\text { Forward } \vec{C}_{i} \leq \sqrt{m_{i}} ; \frac{\tilde{\mathcal{P}}_{i, j}}{\sum_{k=0}^{m_{i}} \widetilde{\mathcal{P}}_{i, k}} \geq \frac{1}{m_{i}} ; \forall n_{j} \in \mathbb{N}_{i} \\
\text { Drop } \quad \vec{C}_{i}>\sqrt{m_{i}} ; \frac{\tilde{\mathcal{P}}_{i, j}}{\sum_{k=0}^{m_{i}} \tilde{\mathcal{P}}_{i, k}}<\frac{1}{m_{i}} ; \forall n_{j} \in \mathbb{N}_{i}
\end{array}\right.
$$

Down-Flow Action: The downlink action, which must be executed on the packets (i.e., drop, forward), is defined by Eq. (17) where $\hat{C}_{i}$ is the threshold value of downlink candidates that are allowed to be selected by the node $n_{i}$.

$$
\overleftarrow{\operatorname{Act}}\left(n_{i}\right)=\left\{\begin{array}{l}
\text { Forward } \overleftarrow{C}_{i} \leq \sqrt{m_{i}} ; \frac{\widetilde{\mathcal{P}}_{i, j, t}}{\sum_{k=0}^{m_{i}} \tilde{\mathcal{P}}_{i, k, t}} \geq \frac{1}{m_{i}} ; \forall n_{j} \in \mathbb{N}_{i} \\
\text { Drop } \quad \overleftarrow{C}_{i}>\sqrt{m_{i}} ; \frac{\widetilde{\mathcal{P}}_{i, j, t}}{\sum_{k=0}^{m_{i}} \tilde{\mathcal{P}}_{i, k, t}}<\frac{1}{m_{i}} ; \forall n_{j} \in \mathbb{N}_{i}
\end{array}\right.
$$

\subsection{System Parameters}

The flows are controlled through the five system parameters, $H$-exponent $\gamma_{H}, R$-exponent $\gamma_{R}, \mathcal{E}$-exponent $\gamma_{\mathcal{E}}$, L-exponent $\gamma_{L}$ and $D$-exponent $\gamma_{D}$. The uplink flows are controlled through the four parameters, $H$-exponent $\gamma_{H}$, R-exponent $\gamma_{R}$, $\mathcal{E}$-exponent $\gamma_{\mathcal{E}}$ and L-exponent $\gamma_{L}$ while the downlink flows 
are controlled through the three parameters $D$-exponent $\gamma_{D}$, $R$-exponent $\gamma_{R}$ and L-exponent $\gamma_{L}$.

To maximize the network lifetime, the value of $L$-exponent $\gamma_{L}$ is set to be greater than the values of the other parameters in both uplink and downlink flows. L-exponent $\gamma_{L}$ is designed to avoid choosing the nodes with low-energy in each transmission session. Greater value of $L$-exponent $\gamma_{L}$ increases the network lifetime, but it enforces the packets to travel via longer paths as it prefers the nodes with highenergy. In order to avoid loops, the values of $H$-exponent $\gamma_{H}$ and $D$-exponent $\gamma_{D}$ should continuously set to be greater than the value of $R$-exponent $\gamma_{R}$. R-exponent $\gamma_{R}$ induces the packets to be transmitted through shorter transmission distance in each transmission session (single hop) without considering the direction towards the sink nor considering the energy level of the relay nodes. H-exponent $\gamma_{H}$ enforces the packet to reach the sink via the minimum number of hops without considering the energy level of the relay nodes. D-exponent $\gamma_{D}$ and $\mathcal{E}$ - exponent $\gamma_{\varepsilon}$ induce the packets to travel towards the direction of the destination node via a shorter routing distance without considering the energy level of the relay nodes nor considering the transmission distance in each transmission session. The values of system parameters are strongly related to the network density and should be set in accordance with the application specifications and requirements. Considering the duty cycle of the nodes, to counterbalance between the sender waiting time and the duplicate packet, the default values for uplink and downlink flows are obtained by Eq. (18) and Eq. (19), respectively. We assign higher priority for energy parameter in both uplink and downlink flows.

$$
\begin{aligned}
& \overrightarrow{P a r}\left(n_{i}\right)=\left\{\begin{array}{l}
\gamma_{L}=\left(1+\sqrt{m_{i}}\right) \\
\gamma_{H}=\left(1+\sqrt{m_{i}}\right) / 2 \pi \\
\gamma_{\varepsilon}=\left(1+\sqrt{m_{i}}\right) / 2 \pi \\
\gamma_{R}=\left(1+\sqrt{m_{i}}\right) / 3 \pi
\end{array}\right. \\
& \overleftarrow{\operatorname{Par}}\left(n_{i}\right)=\left\{\begin{array}{l}
\gamma_{L}=\left(1+\sqrt{m_{i}}\right) \\
\gamma_{D}=\left(1+\sqrt{m_{i}}\right) / 2 \pi \\
\gamma_{R}=\left(1+\sqrt{m_{i}}\right) / 3 \pi
\end{array}\right.
\end{aligned}
$$

\subsection{Flow Update}

The priority value should be updated periodically based on the remaining energy of nodes. Otherwise, higher priority nodes will exhaust their energy earlier. The controller updates the flows based on the statistics reported by the end nodes. In this work, the controller updates the flows for the end node each time the node loses $5 \%$ of its energy.

\section{ANALYSIS}

The performance of MINI-FLOW is fully related to the number of active candidates (wake-up candidates) selected by the sender node in each hop. Therefore, finding the probability of having $\xi$ awake candidate nodes in each hop is important for evaluation. We assume the nodes are asynchronous and have the same active period of length $t$. The active period can be considered as a continuous interval with randomly selected start and end points. We assume that the starting point of each interval is randomly selected within [0,T]. For a given node $n_{i}$, the maximum number of candidates is set to $c_{i}=\sqrt{m_{i}}$, where $m_{i}$ denotes the number of neighbors for a node $n_{i}$. The probability, denoted by $\phi_{i}(\xi=0)$, that all candidates are in sleep state is equal to the probability of no intersection between the active interval of $n_{i}$ and the active intervals of its candidates. This probability depends on the value of $x$ in three cases. The easy case is when $x \in(t, T-t)$ in which each of the other $c_{i}-1$ intervals has probability of $(T-2 t) / T$ of not intersecting. The second case, when $x \in(0, t)$, then for each, the probability of non-intersection for each of the others is $(T-(x+\mathrm{t})) / T$. The third case, when $x \in(T-t, T)$, then for each, the probability of non-intersection for each of the others is $(x-t) / T$. The probability of zero intersection between $n_{i}$ and its candidates is obtained by summing the three cases as in Eq.(20).

$$
\left\{\begin{array}{l}
f_{1}=\int_{t}^{T-t}\left(\frac{T-2 t}{T}\right)^{c_{i}-1} d x=(T-2 t)\left(1-\left(\frac{2 t}{T}\right)\right)^{c_{i}-1} \\
f_{2}=\int_{0}^{t}\left(\frac{T-(x+t)}{T}\right)^{c_{i}-1} d x=\frac{T}{c_{i}}\left(\left(\frac{T-t}{T}\right)^{c_{i}}-\left(\frac{T-2 t}{T}\right)^{c_{i}}\right) \\
f_{3}=\int_{T-t}^{T}\left(\frac{x-t}{T}\right)^{c_{i}-1} d x=\frac{T}{c_{i}}\left(\left(\frac{T-t}{T}\right)^{c_{i}}-\left(\frac{T-2 t}{T}\right)^{c_{i}}\right) \\
\phi_{i}(\xi=0)=\frac{1}{T}\left(f_{1}+f_{2}+f_{3}\right) ; 0<t \leq \frac{T}{2} ; c_{i} \geq 0 ;
\end{array}\right.
$$

The probability of zero-intersection (i.e., no active candidate) gets higher with a smaller number of candidates. For a fixed length of active period $t, \phi_{i}(\xi=0)$ get higher when the initialization interval $[0, T]$ gets greater. Also, for a fixed initialization interval $[0, T], \phi_{i}(\xi=0)$ get higher with a smaller length of active period $t$.

Likewise, the probability that there is only one active candidate is given by Eq. (21) while the probability that there are more than two active candidates is given by Eq. (22).

$$
\begin{gathered}
\phi_{i}(\xi=1)=\left(c_{i}-1\right)\left(\int_{0}^{t} \frac{x+t}{T}\left(\frac{T-(x+t)}{T}\right)^{c_{i}-2} d x+\int_{t}^{T-t} \frac{2 t}{T}\left(\frac{T-2 t}{T}\right)^{c_{i}-1} d x\right. \\
\left.+\int_{T-t}^{T} \frac{T-x+t}{T}\left(\frac{x-t}{T}\right)^{c_{i}-1} d x\right) \\
\phi_{i}(\xi \geq 2)=1-\left[\phi_{i}(\xi=0)+\phi_{i}(\xi=1)\right]
\end{gathered}
$$

To generalize $\phi_{i}(\xi)$, we assume that $[0, T]$ is normalized to $[0,1]$. The probability that there are exactly $\xi\left(c_{i} \geq \xi>\right.$ 1) active candidates and $c_{i}-\xi$ sleep candidates is given by Eq. (23), where $\lambda_{\xi}=1-t\left(c_{i}-\xi\right)$.

$$
\phi_{i}(\xi)=\left(c_{i}-\xi+1\right)\left(\lambda_{\xi}^{c_{i}}-\lambda_{\xi-1}^{c_{i}}\left(\sum_{u=0}^{\xi-2}\left(\begin{array}{c}
c_{i} \\
u
\end{array}\right)\left(\frac{t}{\lambda_{\xi-1}}\right)^{u}\right)\right)
$$

\subsection{Expected Energy Cost}

The energy cost, $T_{X}(i, j, k)$, for transmitting the packet of size $k$ from $n_{i}$ to $n_{j}$ is formulated by Eq. (24) where $E_{\text {elec }}, \varepsilon_{f s}$, and $\varepsilon_{m p}$ are constants, while $d_{*}=$ $\sqrt{\varepsilon_{f s}} / \varepsilon_{m p}$ is the Threshold Distance in meters, and $d_{i, j}$ is the Euclidean distance between the sender $n_{i}$ and the receiver $n_{j}$ [20].

$$
T_{X}(i, j, k)=\left\{\begin{array}{cc}
\left(k \cdot E_{\text {elec }}\right)+\left(k \cdot \varepsilon_{f s} \cdot d_{i, j}^{2}\right) & d_{i, j}<d_{*} \\
\left(k \cdot E_{\text {elec }}\right)+\left(k \cdot \varepsilon_{m p} \cdot d_{i, j}^{4}\right) & d_{i, j} \geq d_{*}
\end{array}(24)\right.
$$

Likewise, the energy required to receive a packet by $n_{j}$ is formulated by Eq. (25).

$$
R_{X}(j, k)=k . E_{\text {elec }}
$$

We compute the energy cost of each transmission phase, which sums the cost of transmitting and receiving the beacon packets, ACK packets, and data packets/control packet. The energy cost of each transmission phase in 
MINI-FLOW is totally determined by the number of active candidates in each hop. Based on Eq. (24) and Eq. (25), in the worst case (i.e., all candidates are active), the energy cost for transmitting and receiving the beacon packet is computed by Eq. (26) where $c_{i}=\sqrt{m_{i}}$ denotes the maximum number of candidates of $n_{i}, k_{b}$ indicates the size of the beacon packet in bits and $n_{j}$ represents the selected next hop.

$$
\begin{gathered}
T_{B}\left(i, j, k_{b}\right)=T_{X}\left(i, j, k_{b}\right)+\sum_{u=1}^{c_{i}} R_{X}\left(u, k_{b}\right)= \\
k_{b}\left\{\begin{array}{cc}
E_{\text {elec }}+\varepsilon_{f s} \cdot d_{i, j}^{2}+c_{i} \cdot E_{\text {elec }} & d_{i, j}<d_{*} \\
E_{\text {elec }}+\varepsilon_{m p} \cdot d_{i, j}^{4}+c_{i} \cdot E_{\text {elec }} & d_{i, j} \geq d_{*}
\end{array}(26)\right.
\end{gathered}
$$

Likewise, the energy required to send back ACK packets to $n_{i}$ is formulated by Eq. (27), where $k_{a}$ denotes the size of the ACK packet.

$$
\begin{gathered}
T_{A}\left(i, k_{a}\right)=\sum_{u=1}^{c_{i}} R_{X}\left(i, k_{a}\right)+\sum_{u=1}^{c_{i}} T_{X}\left(u, i, k_{a}\right)= \\
k_{a}\left(c_{i} \cdot E_{\text {elec }}+\sum_{u=1}^{c_{i}}\left\{\begin{array}{ll}
E_{\text {elec }}+\varepsilon_{f s} \cdot d_{u, i}^{2} & d_{u, i}<d_{*} \\
E_{\text {elec }}+\varepsilon_{m p} \cdot d_{u, i}^{4} & d_{u, i} \geq d_{*}
\end{array}\right)\right.
\end{gathered}
$$

Accordingly, based on Eq. (26) and Eq. (27), the energy cost of each transmission phase is computed by Eq. (28).

$\overline{T_{X}}\left(i, j, k, k_{b}, k_{a}\right)=T_{X}(i, j, k)+R_{X}(j, k)+T_{B}\left(i, j, k_{b}\right)+T_{A}\left(i, k_{a}\right)(28)$

However, the worst case does not often occur. Thus, we are interested in finding a close form for the expected energy cost when there are $\xi\left(c_{i} \geq \xi>1\right)$ active candidate(s) assigned for the sender $n_{i}$. Given $\xi$ active candidates, the energy cost for transmitting and receiving a beacon packet is generalized by Eq. (29).

$$
\begin{aligned}
T_{B}\left(i, j, k_{b}, \xi\right)=T_{X}\left(i, j, k_{b}\right) & +\sum_{u=0}^{\xi} R_{X}\left(u, k_{b}\right) \\
& =k_{b}\left\{\begin{array}{ll}
E_{\text {elec }}+\varepsilon_{f s} . d_{i, j}^{2}+\xi . E_{\text {elec }} & d_{i, j}<d_{*} \\
E_{\text {elec }}+\varepsilon_{m p} . d_{i, j}^{4}+\xi . E_{\text {elec }} & d_{i, j} \geq d_{*}
\end{array}\right. \text { (29) }
\end{aligned}
$$

In the same way, the energy consumed by sending back the ACKs to node $n_{i}$ is generalized by Eq. (30).

$$
\begin{aligned}
T_{A}\left(i, k_{a}, \xi\right) & =\sum_{u=0}^{\xi} R_{X}\left(i, k_{a}\right)+\sum_{u=0}^{\xi} T_{X}\left(u, i, k_{a}\right) \\
& =k_{a}\left(\xi \cdot E_{\text {elec }}+\sum_{\forall n_{u} \in A_{i}}\left\{\begin{array}{cc}
E_{\text {elec }}+\varepsilon_{f s} \cdot d_{u, i}^{2} & d_{u, i}<d_{*} \\
E_{\text {elec }}+\varepsilon_{m p} . d_{u, i}^{4} & d_{u, i} \geq d_{*}
\end{array}\right)\right. \text { (30) }
\end{aligned}
$$

Accordingly, the energy consumption of each transmission phase is generalized by Eq. (31).

$T_{\text {Phase }}\left(i, j, k, k_{b}, k_{a}, \xi\right)=T_{X}(i, j, k)+R_{X}(j, k)+T_{B}\left(i, j, k_{b}, \xi\right)+T_{A}\left(i, k_{a}, \xi\right)$

Finally, based on Eq. (31), the expected energy cost of each transmission phase is obtained by Eq. (32), where $\phi_{i}(\xi=u)$ is the probability that there are exactly $\xi \geq 1$ active candidates assigned for the sender node $n_{i}$.

$$
T_{\text {hop }}(i, j)=\sum_{u=1}^{\xi} \phi_{i}(\xi=u) \cdot T_{\text {Phase }}\left(i, j, k, k_{b}, k_{a}, u\right)(32)
$$

Based on Eq. (32), the expected energy cost for delivering the data packet through the routing path $\mathbb{P}=$ $\left\{n_{1}, n_{2}, n_{3}, \ldots, n_{\rho}\right\}$ is formulated by Eq. (33) where $\varrho$ denotes the number of nodes in the path $\mathbb{P}$, and $\operatorname{Pr}_{4}(\xi=u)$ is given by Eq. (23).

$$
T_{\text {path }}=\sum_{y}^{\varrho} T_{\text {hop }}(y, y+1)=\sum_{y}^{\varrho-1} \sum_{u=1}^{\xi} \phi_{i}(\xi=u) . T_{\text {Phase }}\left(y, y+1, k, k_{b}, k_{a}, u\right)(33)
$$

\subsection{Average Waiting Times (AWTs)}

As the nodes are duty-cycled, the sender may wait for its forwarders to wake up. The AWTs represent the expected value of times the sender should wait for at least one of its forwarders to wake up to receive the packet. That is to say, the sender waits when it has a zero-active candidate. Thus, the probability of zero active candidate (Eq. (20)) is utilized to obtain the AWTs in one-hop as in Eq. (34). The AWTs along the path $\mathbb{P}=\left\{n_{1}, n_{2}, n_{3}, \ldots, n_{\ell}\right\}$ is formulated by Eq. (35) where $\varrho$ denotes the number of nodes in the path.

$$
\begin{aligned}
\mathcal{A}_{i} & =\frac{1}{T}\left[(T-2 t)\left(1-\left(\frac{2 t}{T}\right)\right)^{c_{i}-1}+\frac{2 T}{c_{i}}\left(\left(\frac{T-t}{T}\right)^{c_{i}}-\left(\frac{T-2 t}{T}\right)^{c_{i}}\right)\right] \\
\mathcal{A}_{\text {path }} & =\sum_{y=0}^{\varrho} \mathcal{A}_{y}=\frac{1}{T} \sum_{y=0}^{e}\left((T-2 t)\left(1-\left(\frac{2 t}{T}\right)\right)^{c_{y}-1}+\frac{2 T}{c_{y}}\left(\left(\frac{T-t}{T}\right)^{c_{y}}-\left(\frac{T-2 t}{T}\right)^{c_{y}}\right)\right)
\end{aligned}
$$

\subsection{Redundant Packets (AxRP)}

When the sender node broadcasts a beacon packet, all its awoken candidates will hear the packet and receive it. However, after coordination, one candidate will be selected to forward the packet while the other candidates will abort the received packet. The aborted packets are considered as redundant. AxRP is the average number of aborted packets.

$$
\begin{gathered}
\ddot{\mathcal{W}}_{\text {hop }}=\sum_{u=2}^{\xi} u \cdot \phi_{i}(\xi=u) \\
\ddot{\mathcal{W}}_{\text {path }}=\sum_{y=1}^{\varrho} \ddot{\mathcal{W}}_{\text {hop }}=\sum_{y=1}^{\varrho} \sum_{u=2}^{\xi} u \cdot \phi_{i}(\xi=u)
\end{gathered}
$$

The probability of no redundant packets is obtained by $\phi_{i}(\xi=1)$, Eq. (21), while the probability of redundant packets is obtained by Eq. (22). The expected number of redundant packets in each hope is formulated by Eq. (36) while the expected number of redundant packets along the path $\mathbb{P}=\left\{n_{1}, n_{2}, n_{3}, \ldots, n_{\varrho}\right\}$ is given by Eq. (37).

\section{SIMULATION AND RESULTS 6.1 Simulation Settings}

We evaluated the performance of the proposed protocol using the simulator [28] [29] which is developed in visual studio 2015 (C\# WPF). The documentations of the simulator are available online in the link ${ }^{4}$. We assumed that the nodes are randomly deployed in a fair distribution, and the sink node is placed in the center of the sensing field. For simplicity, at the simulation level, the controller is located in the sink node. We utilized one sink and one controller. Each node runs BOX-MAC. The nodes have the same active (1s)/sleep (2s) periods. Each node has a battery of 0.5 Jouls and consumes energy according to the First Order Radio Model as in [20]. We employed free space propagation model that assumes the ideal propagation condition by representing the communication range as a circle around the transmitter. The packet generation rate is adjusted to $1 / 0.1$ s (one packet in 0.1 second). In each $0.1 \mathrm{~s}$, a random node generates a packet and routes it towards the sink. The 
size of the data packet is 1024bits while the size of the control packet is 512 bits.

TABLE 4: SIMULATION PARAMETERS

(A)
\begin{tabular}{|l|l|}
\hline Parameter & Value \\
\hline Packet Rate & $1 / 0.1 \mathrm{~s}$ \\
\hline Simulation Time & $480 \mathrm{~s}$ \\
\hline Active Time & $1 \mathrm{~s}$ \\
\hline Sleep Time & $2 \mathrm{~s}$ \\
\hline Number of Nodes & 100 \\
\hline
\end{tabular}

(B)
\begin{tabular}{|l|l|}
\hline Parameter & Value \\
\hline Packet Rate & $1 / 0.1 \mathrm{~s}$ \\
\hline Simulation Time & $300 \mathrm{~s}$ \\
\hline Active Time & $1 \mathrm{~s}$ \\
\hline Sleep Time & $2 \mathrm{~s}$ \\
\hline $\mathrm{CR}$ & $80 \mathrm{~m}$ \\
\hline
\end{tabular}

\begin{tabular}{|l|l|}
\hline \multicolumn{2}{|c}{$(\mathrm{C})$} \\
\hline Parameter & Value \\
\hline Packet Rate & $1 / 0.1 \mathrm{~s}$ \\
\hline Simulation Time & $300 \mathrm{~s}$ \\
\hline Sleep Time & $2 \mathrm{~s}$ \\
\hline $\mathrm{CR}$ & $80 \mathrm{~m}$ \\
\hline Number of Nodes & 100 \\
\hline
\end{tabular}

Comparisons to the State-of-the-art: Two well-known WSN protocols are selected:

(1) ORR, a load-balanced opportunistic routing protocol [21]. ORR runs in two steps. Step 1: the optimal number of candidates is computed based on a cost estimation function, which is derived from duty-cycle and network density. Step 2: the residual energy is used during the selection of forwarders [21]. During the simulation experiments, we set the alpha parameter to 1.0. The authors of ORR evaluated ORR by varying the alpha parameter from 0.0 to 4.0 .

(2) ORW, a practical opportunistic routing protocol [22]. ORW utilized the Expected Duty Cycled wakeups (EDC) as a global routing metric. EDC computes the expected number of wakeups until the packet reaches its intended destination (via single hop or multiple hops). Explicitly, EDC defines the number of wakeups as the sum of three terms. First, the expected time needed to forward the packet (one hop). Second, the time needed to forward the packet from the selected forwarder to the intended destination. Third, a small constant accounting the cost of forwarding the packet. These two protocols are implemented using C\# WPF Visual Studio 2015 in the link 5.

\subsection{Simulation Results}

Evaluation Metrics: We considered the following evaluation metrics. (1) Energy Consumption (EC): The total energy consumption required to deliver the packets from the sources to the destination at a given simulation time. The energy unit is Joule. (2) Average Number of Redundant Packets (AxRP): When the sender node broadcasts a packet, all its awoken candidates will hear the packet and receive it. However, after coordination, one candidate will be selected to forward the packet while the other candidates will abort the received packet. The aborted packets are considered as redundant. AxRP is the average number of the aborted packets at a given simulation time. (3) Average Routing Distance Efficiency (RDE): The Routing Distance Efficiency (RDE), measures the ratio of the Euclidean Distance $d_{s, b}$ (between the sources to the sink) to the actual Routing Distance $d_{b}^{s}$ for a data packet that travelled along the path. $R D E=d_{s, b} / d_{b}^{s}$. (4) Average Waiting Times $(A W T)$ : The average number of times the sender waits until one of its potential forwarders wakes up and receives the packet at a given simulation time. (5) Network Lifetime: The time (in seconds) from the starting moment of the simulation to the moment that the first node runs out of energy.

\subsubsection{Varying the Communication Ranges}

This section evaluates the performance of the network varying the communication range. The communication range (CR) varies from $50 \mathrm{~m}$ to $100 \mathrm{~m}$. The default parameters for this experiment are listed in Table 4(A).

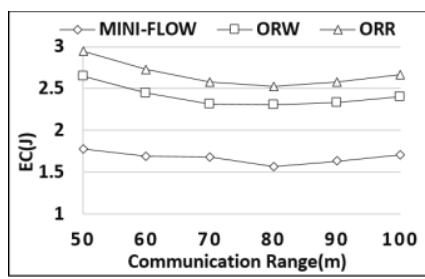

Figure 11: Energy consumption vs. varying communication range.

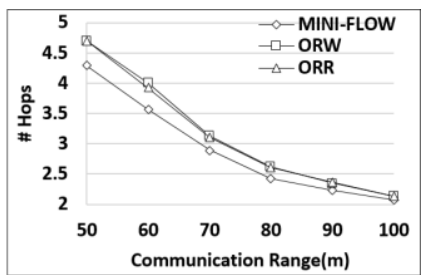

Figure 12: Average number of hops vs. varying communication range. (a) Energy Consumption: The results of evaluating the energy consumption (EC) are depicted in Figure 11. Several observations are concluded. In the case when the communication range is lower than $80 \mathrm{~m}$, the energy consumption decreases as the range of communication increases. The reason behind that is the number of hops decreases as the range of communication gets larger (see Figure 12). However, greater communication range increases the number of candidates for each node, which in turn increases the number of redundant packets (AxRP) and maximizes the energy consumption (see Figure 13). In the case when the communication range is greater than $80 \mathrm{~m}$, the energy consumption increases as the range of communication increases. The reason behind that is the dramatic increment in the negative effect of the duplicate packets AxRP generated from multiple receivers. MINI-FLOW achieved better energy saving than ORR and ORW due to the following two reasons. First, the number of hops is controlled by the H-distribution such that the packets are mostly routed via the paths with the minimal number of hops. Second, the MINI-FLOW forwarders are well controlled by Eq. (16) and Eq. (17) such that few of the nodes are allowed to be selected as forwarders, which in turn reduces the negative effect of the duplicate packets generated from multiple receivers. Although ORR calculates the maximum number of forwarders, it incurs a big amount of energy consumption during the calculation. Moreover, ORR periodically updates the EDC metric to achieve energy balancing among the nodes. However, its EDC metric is recursively calculated in the sink. This means that in each update phase, each node sends its current energy state to the sink that re-computes the EDC metric and sends back the new EDC metric (Expected Duty Cycled) to each node. Obviously, this hinders the network's performance and depletes its restricted resources. ORW computes its EDC metric in the initializing process without considering the residual energy of the nodes. Both ORR and ORW suffer from the negative impact of duplicate packets.

(b) The number of hops: The results of evaluating the number of hops are depicted in Figure 12, which indicate that the number of hops decreases as the range of communication increases since fewer numbers of hops means shorter Routing Distance, which in turn costs less energy. 
The average number of hops in ORW is comparable to ORR since both of them utilize the Expected Duty Cycled wakeups (EDC) as a global routing metric.

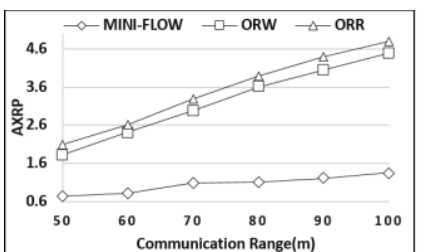

Figure 13: Average number of redundant packets (AxRP) vs. varying communication range.

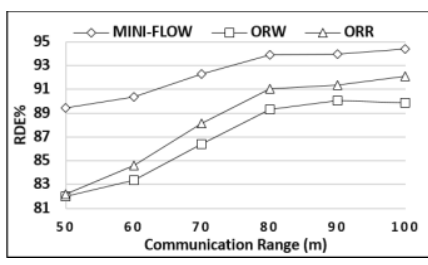

Figure 14: Average Routing Distance Efficiency (RDE) vs. varying communication range.

EDC utilizes the expected number of wakeups until the packet reaches its intended destination (through single hop or multiple hops). ORR is approximately similar to ORW with a minor difference. ORR removes the third term in ORW (i.e., removes the forwarding-cost term from EDC) and adds the residual energy parameter to the first term of EDC. In MINI-FLOW, the number of hops is controlled by the $\mathrm{H}$-distribution such that the packets are regularly routed through minimum hops.

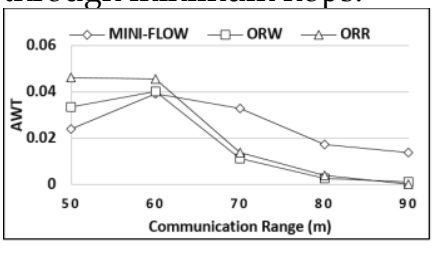

Figure 15: Average waitingtime vs. varying communica-

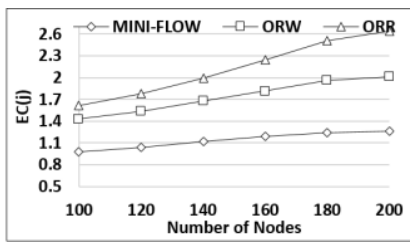

Figure 16: Energy consumption vs. varying number of nodes. (c) Redundant Packets: The results of evaluating the average number of redundant packets (AxRP) are depicted in Figure 13. AxRP measures the negative impact of duplicate packets generated from multiple receivers. We concluded that AxRP increases as the range of communication increases. Greater communication range inevitably imposes the node to select more candidates, which in turn increases the probability of multiple receivers. MINI-FLOW is designed to reduce the number of duplicate packets by Eq. (16) and Eq. (17) in each node such that the number of candidate nodes is controlled by the heuristic function of uplink flows and the heuristic function of downlink flows.

(d) Routing Distance Efficiency: The results of evaluating the average Routing Distance Efficiency (RDE) are depicted in Figure 14. We concluded that for a path, the RDE gets higher as the communication range increases since the packets travel through a smaller number of hops. MINIFLOW shows higher RDE than ORR and ORW because the packets travel through the shortest path to the destination. ORR and ORW selected more forwarder nodes, which in some cases selected the active next hop node even if it is not towards the direction of the destination. This obviously increases the Routing Distance and consequently reduces the RDE.

(e) Average Waiting Times: The results of evaluating the Average Waiting Times (AWT) are shown in Figure 15, from which we concluded that for each path, the AWT gets smaller as the communication range increases. Greater communication range drives the node to employ more candidate nodes, which in turn reduces the waiting time. In fact, increasing the number of candidate nodes has two different impacts, positive and negative. The negative impact is the increment in duplicate packets, which raises the consumption of energy. In contrast, the positive impact is the reduction in the number of waiting times such that the sender needs not to wait for a specific candidate to wake up and receive the packet. These two contradictory impacts are reflected by the nature of asynchronous duty-cycled WSN. From the network layer's point of view, WSN designers utilize multiple candidate nodes to reduce the waiting times. However, too many candidate nodes may simultaneously wake up, causing the duplication problem. MINI-FLOW is designed to counterbalance the two impacts by trading off energy and waiting time.

\subsubsection{Varying the Number of Nodes}

This section evaluates the performance of the network varying the number of nodes. The default parameters are listed in Table 4(B). The number of nodes varies from 100 to 200 .

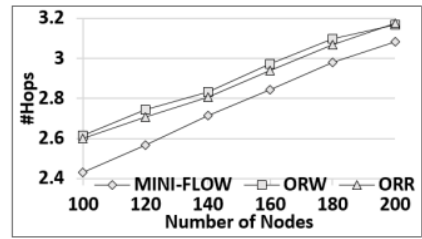

Figure 17: Average number of hops varying a number of nodes.

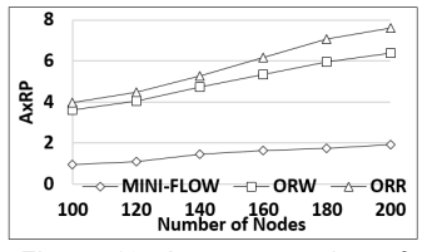

Figure 18: Average number of redundant packets $(A x R P)$ varying number of nodes. (a) Energy Consumption: Figure 16 shows the results of evaluating the energy consumption by varying the number of nodes. In view of that, we concluded that the energy consumption increases as the number of nodes increases. Increasing the number of nodes either leads to a high density or to a large size of the network. High network density increases the number of candidates assigned for each node, which in turn generates more Redundant Packets (AxRP) and consumes more energy. On the other hand, the large size of the network increases the number of hops and forces the packet to travel through longer paths, which in turn consumes more energy. MINI-FLOW outperforms ORR and ORW for the same reasons explained in Subsection 6.2.1(a).

(b) Number of hops: Figure 17 shows the evaluation results of the number of hops varying the number of nodes. We concluded that the number of hops increases as the size of the network increases. MINI-FLOW outperforms ORR and ORW for the same reasons explained in Subsection 6.2.1(b).

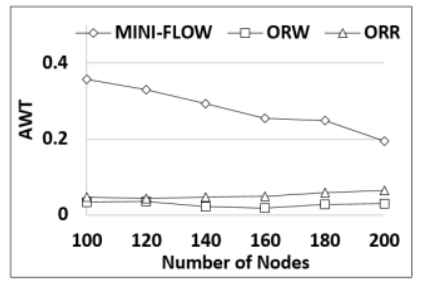

Figure 19: Average waitingtime vs. varying number of nodes.

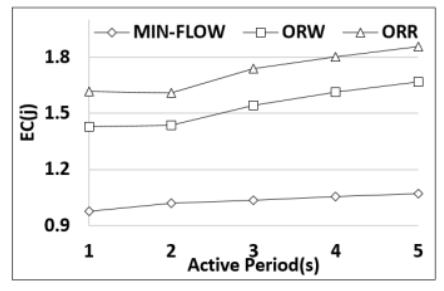

Figure 20: Energy consumption vs. varying active periods. (c) Redundant Packets: Figure 18 shows the results of 
evaluating the redundant packets (AxRP) by varying the number of nodes. We concluded that AxRP increases as the number of nodes grows, since higher network density always leads to a rise in the number of candidates that are assigned for each node. MINI-FLOW outperforms ORR and ORW for the same reasons explained in Subsection 6.2.1(c).

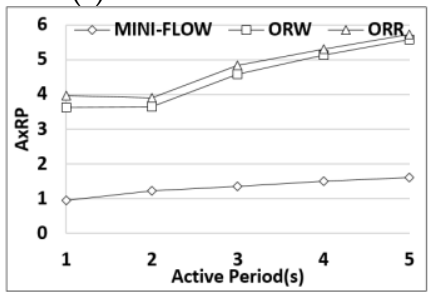

Figure 21: Average number of redundant packets $(A x R P)$ active periods.

(d) Average Waiting Times: The results of evaluating the Average Waiting Times (AWT) are shown in Figure 19, from which we concluded that for each path, the AWT gets smaller as the network density increases. Greater density imposes the node to employ more candidate nodes, which in turn reduces the waiting time. For more details, refer to Subsection 6.2.1(e).

\subsubsection{Varying the Wake-up Intervals}

This section evaluates the performance of the network varying the wake-up intervals. The default parameters are listed in Table 4(C). The active period varies from 1s to 5s.

(a)Energy Consumption: Figure 20 shows the results of evaluating the energy consumption (EC) by varying the active periods. It shows that the longer active periods enforce the nodes to consume more energy for the following two reasons. First, obviously, the node consumes more energy when it stays active for a long time. Second, the longer active periods inevitably increase the probability of multiple receivers. This problem arises when multiple potential forwarders simultaneously wake up and receive the same packet. It definitely degrades the channel capacity, especially when the application requires high traffic load. MINI-FLOW outperforms ORR and ORW for the same reasons explained in Subsection 6.2.1(a).

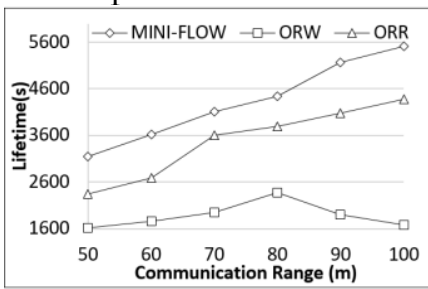

Figure 23: The network lifetime vs. varying communication ranges.

(b) Redundant Packets: Figure 21 shows the evaluation results of redundant packets (AxRP) varying the active periods. It shows that longer active periods generate more AxRP since it undoubtedly increases the probability of multiple receivers. MINI-FLOW outperforms ORR and ORW for the same reasons explained in Subsection 6.2.1(c).

(c) Average Waiting Times: Figure 22 shows the results of evaluating the Average Waiting Times (AWT) varying the active periods. We concluded that for each path, the AWT gets smaller as the active period gets longer. Longer active period drives the node to employ more candidate nodes, which in turn reduces the waiting time. For more details, refer to Subsection 6.2.1(e).

\subsubsection{Network Lifetime}

We evaluate the network lifetime by implementing two scenarios. In the first scenario, different communication ranges are utilized. The number of nodes is set to 100 while the communication range varies from $50 \mathrm{~m}$ to $100 \mathrm{~m}$. The results are plotted in Figure 23. Note that with the fixed area size and number of nodes, larger communication range means higher network density. We concluded that greater communication range implies longer lifetime for two main reasons. First, greater communication range allows the node to have more candidate nodes which in turn enhances the load balancing among them. With higher density, even without employing the load-balancing mechanism, the packets travel throughout diverged paths from the end nodes to the sink or from the controller to the end nodes. Second, greater communication ranges reduce the routing distance, which in turn minimize the energy consumption and maximize the network lifetime. ORW shows the worst performance in terms of the network lifetime since no energy balancing mechanism is considered. Additionally, ORW suffers from the negative impact of duplicated packets. This impact looks more negative when the communication range exceeds $80 \mathrm{~m}$. The Candidate Set in ORW is fixed and computed when the network is initialized which in turn increases the traffic load on nodes with higher EDC. ORR archived longer lifetime than ORW since it defined the maximum number of forwarders. However, to ensure energy balancing, ORR suffers from an expensive cost of periodically updating the EDC metric.

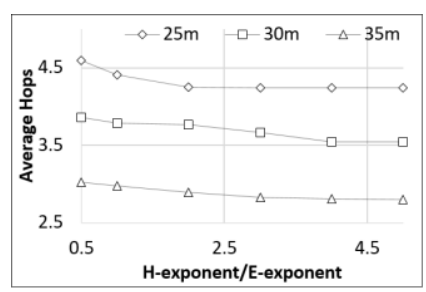

Figure 25: The average number of hops/packet vs. varying the system parameters $\mathrm{H}$-exponent $\gamma_{H}$ and $\mathcal{E}$ - exponent $\gamma_{\mathcal{E}}$

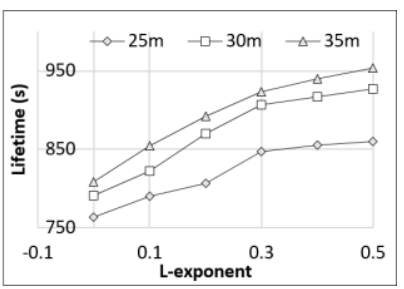

Figure 26: The network lifetime vs. varying the system parameters $L$-exponent $\gamma_{L}$. The initial battery power is $0.1 \mathrm{~J}$.
To update the EDC metric, ORR collects the energy state of all nodes and then re-computes the EDC. MINI-FLOW outperforms ORR and ORW in terms of the network lifetime since L-Distribution enforces the packets to travel through the nodes with higher remaining energy and $R$ Distribution enforces the packets to travel through shorter transmission distance in each transmission session while the three distributions H-Distribution, D-Distribution and $\mathcal{E}$ Distribution enforce the packets to travel via shorter routing distance. Moreover, MINI-FLOW updates the flows based on the node's request. This is different from ORR in which each update session requires the data of the entire network. ORR considered the energy balancing mechanism and neglected the importance of the routing distance of the whole path and the transmission distance in each 
hop.

The second scenario evaluates the lifetime of the network by varying the duty cycles. The number of nodes is set to 100 with $80 \mathrm{~m}$ of communication range. The node sleeps for $2 \mathrm{~s}$ while its active period varies from $1 \mathrm{~s}$ to $5 \mathrm{~s}$. The results are plotted in Figure 24. We concluded that the network lifetime is shorter when the active period of the nodes is longer. This eventuates since longer active periods increase the negative impact of multiple receivers and hence a greater number of redundant packets $(\mathrm{AxRP})$ is generated.

\subsubsection{Impact of System Parameters}

(a) $H$-exponent $\gamma_{H}$ and $\mathcal{E}$-exponent $\gamma_{\mathcal{E}}$. We evaluated the average number of hops by varying the system parameters $\gamma_{H}$ and $\gamma_{\mathcal{E}}$. The values of $\boldsymbol{\gamma}_{\mathcal{E}}$ and $\boldsymbol{\gamma}_{\boldsymbol{H}}$ are varied from 0.5 to 5 such that in each experiment the value of $\gamma_{\varepsilon}$ is equal to the value of $\gamma_{H}$ while the other system parameters are fixed, $\gamma_{L}=\gamma_{R}=0.1$ and $\gamma_{D}=0.4$. We used three networks each has 100 nodes and the communication radius is set to $25 \mathrm{~m}$ $30 \mathrm{~m}$ and $35 \mathrm{~m}$. Each node sends 1packet/s until the first node runs out of energy. The initial battery power is set to 0.1J. The results are shown in Figure 25. We concluded that the average number of hops is reduced when the values of $H$-exponent $\boldsymbol{\gamma}_{\boldsymbol{H}}$ and $\mathcal{E}$-exponent $\boldsymbol{\gamma}_{\mathcal{E}}$ increase since these two parameters prioritize the node that has a smaller number of hops and a shorter routing distance.

(b) L-exponent $\gamma_{L}$. This subsection evaluates the network lifetime by varying the value of $\gamma_{\mathbf{L}}$, from 0.0 to 0.5 . The values of the other parameters are fixed, $\gamma_{H}=\gamma_{R}=0.1$ and $\gamma_{\varepsilon}=\gamma_{D}=0.5$. We used three networks each has 100 nodes while the communication radius is set to $25 \mathrm{~m}, 30 \mathrm{~m}$ and $35 \mathrm{~m}$. Each node sends 1packet/s until the first node runs out of energy. The results are shown in Figure 26. We concluded that the network achieves longer lifetime as the value of $L$-exponent $\gamma_{L}$ increases since it prioritizes the nodes with higher energy. However, the impact of L-exponent $\gamma_{L}$ gets smaller when its value gets larger than 1.0. $L$ exponent $\gamma_{L}$ enhances load balancing as long as its value is approximately equal to Eq. (18) and Eq. (19). Otherwise, it constructs longer paths in order to avoid low-energy nodes, which in turn maximizes the average packet delay and increases the average number of hops.

\subsubsection{Controller to End Nodes Overhead}

This subsection evaluates the communication overhead of updating the flows varying network size. That is the energy consumed for transmitting the control packets from the controller to the end nodes. This overhead is intensely related to flow-update-loss-percentage (FULP), denoted by $\sigma$. The controller updates the flows of the end node each time the node loses $\sigma \%$ of its energy. We ignored the overhead of network initialization. The network size varies from $300 \mathrm{~m}^{2}$ to $500 \mathrm{~m}^{2}$ while $\sigma$ varies from 5 to 15 . The communication range is set to $50 \mathrm{~m}$, while the number of nodes increases with the size of the network. We set the initial battery power to $0.1 \mathrm{~J}$ and compute the percentage of the energy consumed for transmitting the control packets from the controller to the end nodes. The results are shown in Figure 27. Two observations are concluded. First, greater network sizes increase the communication overhead. Second, greater values of $\sigma$ increase the overhead too.

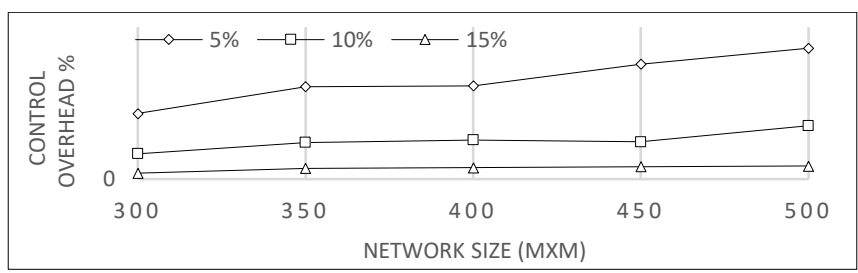

Figure 27: The communication overhead of control packets varying the network size and FULP

\section{CONCLUSION}

This paper investigates the software-defined wireless sensor networks and proposes an architecture and incorporated protocol to enhance the efficiency and scalability. The proposed architecture, MINI-SDN, is an attempt towards separating the control logic from the sensor nodes/actuators. In fact, complete separation of the two planes may degrade the performance of WSNs since such networks are intrinsically distributed and data centric. We proposed a data-centric protocol called MINI-FLOW that supports centralized and distributed routing mechanisms. MINI-FLOW presents three routing mechanisms, the uplink, downlink and intra-link. The uplink routing is based on a heuristic function that combines four values, the number of hops to the sink, the distance towards the sinks, the Received Signal Strength and the remaining energy. Regarding the downlink flows, we proposed two heuristic algorithms. The first algorithm, Optimized Reverse Downlink, considers the reverse paths of uplink flows. The second algorithm, Location-based Downlink, computes the paths based on a heuristic function that combines three values, distance to the end node, the remaining energy and RSS value. The experimental results show our architecture and protocol scale well with both network size and density.

\section{ACKNOWLEDGMENTS}

This paper is supported by the "Fundamental Research Funds for the Central Universities NO. WK2150110012, WK2150110007" and by the National Natural Science Foundation of China (NO. 61772490, 61472382, 61472381 and 61572454).

\section{REFERENCES}

[1] Hyojoon Kim, and Nick Feamster. "Improving network management with software defined networking." IEEE Communications Magazine 51, no. 2 (2013): 114-119.

[2] Deze Zeng, Toshiaki Miyazaki, Song Guo, Tsuneo Tsukahara, Junji Kitamichi, and Takafumi Hayashi. "Evolution of software-defined sensor networks." In Mobile Ad-hoc and Sensor Networks (MSN), 2013 IEEE Ninth International Conference on, pp. 410-413. IEEE, 2013.

[3] Laura Galluccio, Sebastiano Milardo, Giacomo Morabito, and Sergio Palazzo. "SDN-WISE: Design, prototyping and experimentation of a stateful SDN solution for WIreless SEnsor networks." In Computer Communications (INFOCOM), 2015 IEEE Conference on, pp. 513-521. IEEE, 2015.

[4] Salvatore Costanzo, Laura Galluccio, Giacomo Morabito, and Sergio Palazzo. "Software defined wireless networks: Unbridling SDNs." In Software Defined Networking (EWSDN), 2012 European Workshop on, pp. 1-6. IEEE, 2012.

[5] Israat Tanzeena Haque, and Nael Abu-Ghazaleh. "Wireless software defined networking: A survey and taxonomy." IEEE Communications Surveys $\mathcal{E}$ Tutorials 18 , no. 4 (2016): 2713-2737. 
[6] Musa Ndiaye, Gerhard P. Hancke, and Adnan M. Abu-Mahfouz. "Software defined networking for improved wireless sensor network management: A survey." Sensors 17, no. 5 (2017): 1031.

[7] Kgotlaetsile Mathews Modieginyane, Babedi Betty Letswamotse, Reza Malekian, and Adnan M. Abu-Mahfouz. "Software defined wireless sensor networks application opportunities for efficient network management: A survey." Computers E Elect.Engineering, vol. 66 (2018): 274-287.

[8] Hlabishi I. Kobo, Adnan M. Abu-Mahfouz, and Gerhard P. Hancke. "A survey on software-defined wireless sensor networks: Challenges and design requirements." IEEE Access5 (2017): 1872-1899.

[9] Tie Luo, Hwee-Pink Tan, and Tony QS Quek. "Sensor OpenFlow: Enabling software-defined wireless sensor networks." IEEE Communications letters 16, no. 11 (2012): 1896-1899.

[10] Alejandro De Gante, Mohamed Aslan, and Ashraf Matrawy. "Smart wireless sensor network management based on software-defined networking." In Communications (QBSC), 2014 27th Biennial Symposium on, pp. 71-75. IEEE, 2014.

[11] David Moss, and Philip Levis. "BoX-MACs: Exploiting physical and link layer boundaries in low-power networking." Computer Systems Laboratory Stanford University (2008): 116-119.

[12] Yogita Chapre, Prasant Mohapatra, Sanjay Jha, and Aruna Seneviratne. "Received signal strength indicator and its analysis in a typical WLAN system (short paper)." In Local Computer Networks (LCN), 2013 IEEE 38th Conference on, pp. 304-307. IEEE, 2013.

[13] Diego Kreutz, Fernando MV Ramos, Paulo Esteves Verissimo, Christian Esteve Rothenberg, Siamak Azodolmolky, and Steve Uhlig. "Software-defined networking: A comprehensive survey." Proceedings of the IEEE 103, no. 1 (2015): 14-76.

[14] Ammar Hawbani, Xingfu Wang, Saleem Karmoshi, Hassan Kuhlani, Aiman Ghannami, Adili Abudukelimu, and Rafia Ghoul. "GLT: Grouping Based Location Tracking for Object Tracking Sensor Networks." Wireless Communications and Mobile Computing 2017 (2017).

[15] Miroslav Botta, and Milan Simek. "Adaptive distance estimation based on RSSI in 802.15. 4 network." Radioengineering 22, no. 4 (2013): 1162-1168.

[16] Toshiaki Miyazaki, Shoichi Yamaguchi, Koji Kobayashi, Junji Kitamichi, Song Guo, Tsuneo Tsukahara, and Takafumi Hayashi. "A software defined wireless sensor network." In Computing, Networking and Communications (ICNC), 2014 International Conference on, pp. 847-852. IEEE, 2014.

[17] Jihoon Kang, Daeyoung Kim, and Youngsoo Kim. "RSS self-calibration protocol for WSN localization." In Wireless Pervasive Computing, 2007. ISWPC'07. 2nd International Symposium on. IEEE, 2007.

[18] Ben Pfaff, B. Heller, D. Talayco, D. Erickson, G. Gibb, G. Appenzeller, J. Tourrilhes et al. "OpenFlow Switch Specification." Feb 28 (2011): 1-56.

[19] Nascimento, Vagner, Marcelo Moraes, Rosivaldo Gomes, Billy Pinheiro, Antônio Abelém, Vinicius CM Borges, Kleber V. Cardoso, and Eduardo Cerqueira. "Filling the gap between software defined networking and wireless mesh networks." In Network and Service Management (CNSM), 2014 10th International Conference on, pp. 451-454. IEEE, 2014.

[20] Ammar Hawbani, Xingfu Wang, Saleem Karmoshi, Lin Wang, and Naji Husaini. "Sensors grouping hierarchy structure for wireless sensor network." International Journal of Distributed Sensor Networks 11, no. 8 (2015): 650519.

[21] Jungmin So, and Heejung Byun. "Load-balanced opportunistic routing for duty-cycled wireless sensor networks." IEEE Transactions on Mobile Computing 16, no. 7 (2017): 1940-1955.

[22] Euhanna Ghadimi, Olaf Landsiedel, Pablo Soldati, Simon Duquennoy, and Mikael Johansson. "Opportunistic routing in low duty-cycle wireless sensor networks." ACM Transactions on Sensor Networks (TOSN) 10, no. 4 (2014): 67.

[23] Azzedine Boukerche, and Amir Darehshoorzadeh. "Opportunistic routing in wireless networks: Models, algorithms, and classifications." ACM Computing Surveys (CSUR) 47, no. 2 (2015): 22.

[24] P. Santi "Topology control in wireless ad hoc and sensor networks". ACM computing surveys (CSUR), 37 no. 2 (2005), 164-194.

[25] Wei Xiang, Ning Wang, and Yuan Zhou. "An energy-efficient routing algorithm for software-defined wireless sensor networks." IEEE Sensors Journal 16, no. 20 (2016): 7393-7400.

[26] Deze Zeng, Peng Li, Song Guo, Toshiaki Miyazaki, Jiankun Hu, and Yong Xiang. "Energy minimization in multi-task software-defined sensor networks." IEEE Transactions on Computers 64, no. 11 (2015): 3128-3139.

[27] Guozhi Li, S. Guo, Y. Yang and Y. Yang. "Traffic Load Minimization in Software Defined Wireless Sensor Networks," in IEEE Internet of Things Journal, vol. 5, no. 3 (2018): 1370-1378.
[28] Ammar Hawbani, Xingfu Wang, Adili Abudukelimu, Hassan Kuhlani, Yaser Al-sharabi, Ammar Qarariyah, and Aiman Ghannami. "Zone Probabilistic Routing for Wireless Sensor Networks." IEEE Transactions on Mobile Computing 18, no. 3 (2019): 728-741.

[29] Ammar Hawbani, Xingfu Wang, Yaser Sharabi, Aiman Ghannami, Hassan Kuhlani, and Saleem Karmoshi. "LORA: Load-Balanced Opportunistic Routing for Asynchronous Duty-Cycled WSN." IEEE Transactions on Mobile Computing 18, no. 7 (2018): 1601-1615.

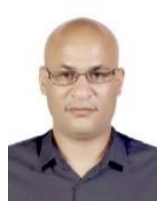

Ammar Hawbani is an associate professor of networking and communication algorithms in the School of Computer Science and Technology at University of Science and Technology of China, China. He received the B.S., M.S. and Ph.D. degrees in Computer Software and Theory from the University of Science and Technology of China (USTC), Hefei, China, in 2009, 2012 and 2016, respectively. From 2016 to 2019, he worked as Postdoctoral Researcher in the School of Computer Science and Technology at USTC. His research interests include IoT, WSNs, WBANs, WMNs, VANETs and SDN.

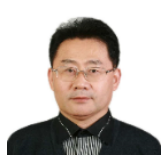

Xingfu Wang received the B.S. degree in electronic and information engineering from Beijing Normal University of China in 1988, and the M.S. degree in computer science from the University of Science and Technology of China in 1997. He is an associate professor in the School of Compute Science and Technology, University of Science and Technology of China. His current research interests include Information Security, Data Management and WSN.

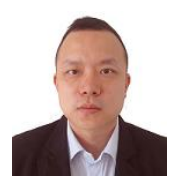

Liang Zhao is a lecturer at Shenyang Aerospace University, China. He received his PhD degree from the School of Computing at Edinburgh Napier University in 2011. Before joining Shenyang Aerospace University, he worked as associate senior researcher in Hitachi (China) Research and Development Corporation from 2012 to 2014 . His research interests include WMNs, VANETs and SDN.

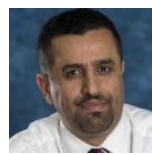

Ahmed ADubai [SM] is Professor of Networking and Communication Algorithms in the School of Computing at Edinburgh Napier University, UK. He received the PhD degree in Computing from the University of Glasgow in 2004. His research interests include Communication Algorithms, Mobile Communication, Internet of Things, and Future Internet.

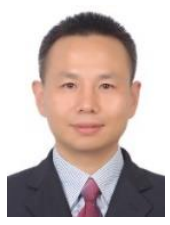

Geyong Min received the Ph.D. degree in computing science from the University of Glasgow, Glasgow, U.K., in 2003. He is a Professor of High Performance Computing and Networking with the Department of Mathematics and Computer Science, University of Exeter, Exeter, U.K. His current research interests include future Internet, wireless communications, multimedia systems, information security, high performance computing, ubiquitous computing, modeling, and performance engineering.

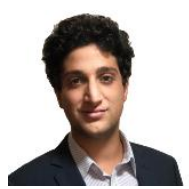

Omar Busaileh received his B.S. degree in Electronic and Information Engineering from Hefei University of Technology. Currently a Master student in the School of Computer Science and Technology at USTC. His research interests mainly include WSNs, WBANs and SDN. 